Ina Berninger, Tim Schröder*

\title{
Inklusion oder Schließung? Gewerkschaftlicher Organisationsgrad, berufliche Geschlechtersegregation und der Gender Pay Gap**
}

\section{Zusammenfassung}

Der Beitrag beleuchtet den Zusammenhang von gewerkschaftlichem Nettoorganisationsgrad (NOG) und dem Gender Pay Gap (GPG) in segregierten Berufen. Auf Basis der Daten des Soziooekonomischen Panels wird unter Anwendung von Mehrebenenmodellen untersucht, ob und in welchen Berufstypen - Männer-, Frauen- oder Mischberufen - der NOG in Branchen-Berufs-Zellen zur Inklusion von Frauen und somit zur Reduzierung des GPG beiträgt oder ob von sozialer Schließung auszugehen ist, der GPG sich also mit dem NOG erhöht. Die Ergebnisse deuten auf eine Inklusion von Frauen hin. Dies trifft jedoch vor allem auf die Frauen- und Mischberufe zu. In Männerberufen ist der GPG zwar insgesamt niedriger und beide Geschlechter können mit steigendem NOG Lohnzuwächse erzielen, der GPG reduziert sich jedoch nicht mit steigendem NOG.

\section{Inclusion or closure? Unionisation, occupational segregation and the gender pay gap}

\section{Abstract}

The article examines the relationship between trade union net density and the gender pay gap (GPG) in segregated occupations. Based on data from the German Socio-Economic Panel and with the use of multilevel models we ask whether and in what job types - male, female or mixed occupations - trade unions pursue inclusion of women and thus contribute to reducing the GPG or whether social closure is prevalent, so that the GPG increases with unionisation. The findings point to an inclusion of women. However, this applies especially to female and mixed occupations. In male occupations, although the GPG is lower overall and both sexes achieve wage increases with increasing unionisation, the GPG is not reduced.

Keywords: Gender Pay Gap, Trade Unions, Segregation

* Dr. rer. pol. Ina Berninger, Universität zu Köln, Institut für Soziologie und Sozialpsychologie, D-50939 Köln. E-Mail: berninger@wiso.uni-koeln.de

Tim Schröder, Universität Hamburg, Fakultät für Wirtschafts- und Sozialwissenschaften, D-20146 Hamburg E-Mail: tim.schroeder@wiso.uni-hamburg.de

** Artikel eingegangen: 26.4.2016, revidierte Fassung akzeptiert nach doppelt-blindem Begutachtungsverfahren: 30.11.2016 


\section{Einleitung}

Trotz eines erheblichen Rückgangs geschlechtsspezifischer sozialer Ungleichheit in Bildung und Erwerbsbeteiligung liegen die Löhne der Frauen in Deutschland im Jahre 2014 etwa 22 Prozent unter denen der Männer (Gartner, 2016). Dieser so genannte Gender Pay Gap (GPG) verharrt seit mehr als 20 Jahren auf ähnlichem Niveau und hat sich seit Beginn der 2000er Jahre sogar leicht erhöht.

Die berufliche Geschlechtersegregation, das heißt die Verteilung von Frauen und Männern auf unterschiedliche Berufe, gilt als bedeutsam bei der Erklärung des GPG. Die zwischen Arbeitsmarkt und betrieblicher Organisation verortete soziale Institution „Beruf“ (Pries, 2010: 31ff) bietet für Frauen und Männer unterschiedliche Opportunitäten und Restriktionen für die Karrieremobilität. Empirische Untersuchungen für Deutschland stellen fest, dass die Beschäftigten in Frauenberufen auch nach Kontrolle der individuellen Humankapitalausstattung niedrigere Löhne erzielen (Kunze, 2005; Busch, 2013; Hausmann, Kleinert \& Leuze, 2015). ${ }^{1}$ Bei den jungen Kohorten sowie beim Arbeitsmarkteintritt verschwindet dieser Einfluss. Jedoch nimmt die innerberufliche Geschlechterungleichheit zu (Aisenbrey \& Brückner, 2008; Kleinert \& Matthes, 2008; Hausmann, Kleinert \& Leuze, 2015). In diesem Zusammenhang wird die Frage aufgeworfen, welche sozialen Mechanismen auf beruflicher Ebene sich hinter dem Einfluss der Geschlechtersegregation auf den GPG verbergen (Liebeskind, 2004; Busch, 2013).

Bislang weitestgehend unerforscht ist hierbei die Rolle der Gewerkschaften (Kleinert \& Leuze, 2015: 235), die im Rahmen kollektiver Lohnverhandlungen zentral an der Lohnfindung beteiligt sind (Schmalz \& Dörre, 2014). Zwar gibt es Untersuchungen, die sich mit dem Einfluss des Nettoorganisationsgrades (NOG) - dem Anteil erwerbstätiger Gewerkschaftsmitglieder an allen Erwerbstätigen - auf die Löhne und die Lohnverteilung beschäftigen. Diese beziehen sich in Deutschland jedoch hauptsächlich auf einen Teil der Arbeitnehmerschaft: männliche Vollzeitbeschäftigte (Fitzenberger, Kohn, \& Lembcke, 2013; Fitzenberger $\&$ Kohn, 2005). Ferner fehlt es der bisherigen Forschung an einer Verknüpfung mit der beruflichen Geschlechtersegregation (Dingeldey, Schröder \& Kathmann, 2015).

Der Beitrag trägt zur Schließung dieser Forschungslücke bei und beleuchtet den Zusammenhang von gewerkschaftlichem NOG und dem GPG in segregierten Berufen mit den Daten des Sozio-oekonomischen Panels. Es wird die Frage erörtert, ob und in welchen Berufstypen - Männer-, Frauen- oder Mischberufen - Gewerkschaften zur Inklusion von Frauen und somit zur Reduzierung des GPG beitragen oder ob von sozialen Schließungsstrategien auszugehen ist, welche den GPG erhöhen. Schließung bedeutet in diesem Zusammenhang, dass Frauen im Allgemeinen und in Frauenberufen im Besonderen aufgrund der historisch männlich geprägten Gewerkschaften im Sinne einer Strategie des Ausschlusses von höheren Positionen benachteiligt werden (Gottschall \& Schröder, 2013; Schröder \& Schäfer, 2013). Die differenzierte Betrachtung nach Berufstypen ermöglicht einen tieferen Einblick in den Mechanismus der Lohnfindung in geschlechtersegregierten Berufen. Es wird die Frage beleuchtet, inwieweit Gewerkschaften ihre Machtressourcen in geschlechtsspezifisch unter-

1 Nach wie vor kommt auch Unterschieden im individuellen Humankapital ein großer Erklärungsanteil zu. Dies gilt weniger für die Bildung als vielmehr die Berufserfahrung (Busch, 2013). Da Frauen häufig in Teilzeit arbeiten und im Anschluss an eine Geburt ihre Erwerbstätigkeit unterbrechen, können sie weniger Humankapital „on-the-job“ akkumulieren. Es ist aber davon auszugehen, dass auch hier die Art des Berufs eine wesentliche Rolle spielt, mithin die Geschlechtersegregation die Berufserfahrung mitbestimmt (Stuth \& Henning, 2014). 
schiedlichem Maße bündeln (können) und somit dazu beitragen, den GPG zu schließen. Die Machtressource des NOG wird dabei definiert als eine nach Branchen und Berufen differenzierte Aggregation der individuellen Gewerkschaftsmitgliedschaft. Dies trägt einerseits der branchenspezifischen Gewerkschaftsorganisation in Deutschland und andererseits der beruflichen Geschlechtersegregation Rechnung.

\section{Theoretische Überlegungen und empirische Befunde}

Das zentrale Ziel von Gewerkschaften als Interessenvertretung der Arbeitnehmerschaft ist es, das primäre Machtgefälle zwischen Kapital und Arbeit (Offe \& Hinrichs, 1977) zu reduzieren (Gottschall \& Schröder, 2013). Im System der abhängigen Lohnarbeit sind die individuellen Beschäftigten darauf angewiesen, ihren Lebensbedarf durch das Anbieten ihrer Arbeitskraft auf dem Markt zu decken, da sie sich nicht im Besitz der Produktionsmittel befinden. Unter der Voraussetzung eher großbetrieblicher Strukturen, d.h. weniger Nachfrager und vieler Anbieter (Oligopsone oder Monopsone), haben Arbeitgeber die Möglichkeit, in individuellen Lohnverhandlungen (implizit) mit der Konkurrenz durch weitere Arbeitskräfte zu drohen und die Löhne so zu reduzieren. Benachteiligt werden Beschäftigte mit geringer individueller Verhandlungsmacht. Historisch trifft dies eher auf die Arbeiterschaft und weniger auf Beamte und die ursprünglich als Privatbeamte bezeichneten höheren Angestellten zu (ebd.). Durch kollektive Organisation und die Aushandlung von Löhnen und Arbeitsbedingungen, die sich in Tarifverträgen manifestiert, verschafft sich die Arbeiterschaft einen Anteil am Mehrwert der Arbeit, der über jenen Anteil hinausgeht, den die individuellen Arbeiter in der Summe erwirtschaften würden (Schmalz \& Dörre, 2014).

In Bezug auf den Einfluss der Gewerkschaften werden zumeist zwei Maße unterschieden: a) der gewerkschaftliche (Netto-)Organisationsgrad (NOG) als Anteil erwerbstätiger Gewerkschaftsmitglieder an allen Erwerbstätigen sowie b) die Tarifbindung als Anteil von Erwerbstätigen, in deren Betrieb ein Tarifvertrag gilt, an allen Erwerbstätigen (Ebbinghaus \& Kittel 2006; Fitzenberger, Kohn \& Lembcke, 2013). ${ }^{2}$ Im internationalen Vergleich wird deutlich, dass beide Maße nur lose miteinander zusammenhängen (Ebbinghaus \& Kittel 2006). Während in liberalen Marktdemokratien, wie Großbritannien und den USA, NOG und Tarifbindung schwach ausgeprägt sind, besteht in Deutschland trotz eines im Vergleich zu den skandinavischen Ländern relativ geringen NOG eine hohe Tarifbindung. Bispinck und Schulten (2011) führen dies primär auf die Rolle des Staates zurück: Erstens besteht das Recht der negativen Koalitionsfreiheit, gemäß dem Nicht-Gewerkschaftsmitglieder nicht diskriminiert werden dürfen und somit auch von Tarifverträgen gedeckt sind. Zweitens sorgt der Staat als Arbeitgeber sowie öffentlicher Auftraggeber für die Einhaltung von Tarifstandards. So weisen Frauen trotz eines geringeren NOG aufgrund der Branchen- und Betriebsstruktur, vor allem der Beschäftigung im öffentlichen Dienst, eine leicht höhere Tarifbindung auf als Männer (Biebeler, 2013; Amlinger \& Bispinck, 2013). Das Instrument der Allgemeinverbindlichkeit spielt hingegen eine quantitativ eher geringe Rolle, nicht zuletzt aufgrund der Vetomacht der Arbeitgeberverbände. Dennoch tragen auch die Arbeitgeber zum hohen Deckungsgrad von Tarifverträgen in Deutschland bei. Sie weisen einen relativ ausgeprägten Organisationsgrad auf und haben - gemäß dem Modell der „Lohnführerschaft“ zumindest in industriellen Schlüsselindustrien - durchaus ein Interesse an der Ordnungs-

2 Zur Diskussion der Gewerkschaftsmacht und weiterer Maße siehe Hirsch und Schnabel (2014). 
funktion und Transaktionskostenersparnis von Tarifverträgen (Ebbinghaus \& Kittel 2006; Bispinck \& Schulten 2011). Der Tarifbindungsgrad ergibt sich also aus dem Zusammenspiel von Tarifparteien und staatlichen Institutionen. Dabei noch unberücksichtigt bleiben Qualität und Inhalt von Tarifverträgen. Angesichts des starken Rückgangs der Tarifbindung kommt der „Verbetrieblichung der Interessenvertretung im Sinne einer Gewichtsverlagerung von der tariflichsektoralen auf die betriebliche Ebene“ unter anderem in Form der Etablierung von Öffnungsklauseln und Firmentarifverträgen eine zunehmende Bedeutung zu (Schmierl, 2003: 653). Diesbezüglich lassen sich negative Auswirkungen auf die Lohnentwicklung auch in tarifvertraglich gebundenen Betrieben vermuten. Die Tarifbindung, so lässt sich schlussfolgern, ist nur ein ungenaues Maß des Einflusses der kollektiven Interessenvertretung auf die Lohnfindung sowie die Lohnstruktur verschiedener Beschäftigtengruppen.

Der NOG gilt in diesem Zusammenhang als konkrete, direkte Machtressource im Sinne von Organisationsmacht (ASU, 2013; Schmalz \& Dörre, 2014). Zunächst ist die Mitgliedschaft in Gewerkschaften (anders als die Tarifbindung) eine individuelle Entscheidung und somit eng mit Beschäftigteninteressen verbunden. Durch die Aggregation zum NOG in Branchen, Berufen und Betrieben können diese Interessen besser vertreten werden (ASU, 2013: 353). Ein höherer NOG sollte auch die Strategiefähigkeit kollektiver Akteure erhöhen. ${ }^{3} \mathrm{Da}$ sich der NOG als Machtressource also aus den individuellen Mitgliedschaftsentscheidungen ergibt, somit eng mit den Interessen von Beschäftigtengruppen verknüpft und zugleich ein genaueres Maß der Möglichkeiten zur strategischen Organisation dieser Interessen ist, rückt er im Folgenden ins Zentrum. Eine allgemeine These ist dabei, dass Gewerkschaften mit einer höheren Organisationsmacht grundsätzlich, das heißt auch unabhängig von der Tarifbindung, höhere Tariflöhne aushandeln können. Da er, ebenso wie die Tarifbindung, aber keine direkten Rückschlüsse auf die Qualität der Tarifverträge zulässt, stellt auch der NOG zunächst eine bloß quantitative Machtressource dar.

An dieser Stelle kommt eine weitere Machtressource ins Spiel: die strukturelle Macht. Diese Machtressource bezieht sich insbesondere auf „die Stellung der Lohnabhängigen im ökonomischen System“ und kann zunächst auch ohne kollektive Organisation wirksam sein (ASU, 2013: 347). Wir begreifen strukturelle Macht in erster Linie als Marktmacht, die auf zwei eng zusammenhängenden, aber theoretisch klar zu trennenden Faktoren beruht: a) einerseits auf der (beruflichen) Qualifikation und ihrer hiervon abgeleiteten Produktivität; b) andererseits auf einer Monopolstellung von Lohnabhängigen und der Möglichkeit „Renten“ zu generieren, d.h. Löhne zu erzielen, die oberhalb des bei freier individueller Konkurrenz entstehenden Lohnniveaus liegen (Bol \& Weeden, 2015). ${ }^{4}$ Mit Weber (1921[1976]: $201 \mathrm{ff}$.) kann diese „Monopolisierung von Chancen“ als soziale „Schließung“ im Sinne einer

3 Weitere Quellen der Organisationsmacht sind materielle und personelle Infrastrukturressourcen, eine effiziente Organisationsstruktur, mittels derer diese Ressourcen eingesetzt werden können, sowie ein innerer Zusammenhalt der Gewerkschaftsmitglieder, der etwa in einer Kollektividentität zum Ausdruck kommt (ASU, 2013: 353ff.).

4 Die Produktionsmacht, das heißt die konkrete Möglichkeit, den betrieblichen Produktionsprozess zu stören (ASU, 2013: 347f), begreifen wir als abgeleitet von der Marktmacht. Wir beschränken uns hier zudem auf die organisationalen und strukturellen Machtressourcen, da der Zusammenhang zwischen gewerkschaftlichem Handeln und Schließungsprozessen innerhalb der Lohnabhängigen im Vordergrund steht. Darüber hinaus werden in der Literatur gesellschaftliche und institutionelle Machtressourcen unterschieden (ASU, 2013). Mit gesellschaftlicher Macht ist der Grad der Einbeziehung in die Gesellschaft gemeint, der durch den Zusammenschluss mit anderen gesellschaftlichen Akteuren entsteht, wodurch der gewerkschaftliche Handlungsspielraum vergrößert wird. Die institutionelle Macht bezieht sich auf erlangte Rechte, wie beispielsweise das Streikrecht oder Institutionen wie das Arbeitsgericht. 
Beschränkung des Wettbewerbs mit den Ausgeschlossenen bezeichnet werden. Sie vollzieht sich gerade nicht auf Grundlage von Produktivitätsunterschieden, sondern durch ,irgendein äußerlich feststellbares Merkmal eines Teils der (aktuell oder potentiell) Mitkonkurrierenden“. Die Einbettung des insbesondere von Parkin (1979) ausgearbeiteten Weberschen Schließungsbegriff in den Machtressourcenansatz erlaubt es, sowohl die Klassenpolarisierung zwischen Kapital und Arbeit als auch und vor allem soziale Spaltungen innerhalb der Arbeiterschaft, „Grenzziehungen im Arbeitsmarkt“ (ASU, 2013: 350), zu erfassen.

Von zentraler Bedeutung hier ist der Zusammenhang von strukturellen Machtasymmetrien innerhalb der Lohnarbeiterschaft und Organisationsmacht. Thema dieser Arbeit und theoretisch kaum erfasst ist die Frage, welche Rolle „geschlechtsspezifische Zuschreibungen“ (ebd.) hierbei spielen, das heißt, ob und in welchen Berufstypen - Männer-, Frauen- oder Mischberufen - Gewerkschaften zur Inklusion von Frauen und somit zur Reduzierung des GPG beitragen oder ob von sozialen Schließungsstrategien auszugehen ist, welche den GPG erhöhen. In Bezug auf den allgemeinen Einfluss der Gewerkschaften auf den GPG zeigen sich zwei konkurrierende Strategien, die wir im Folgenden als Inklusions- und Schließungsstrategie bezeichnen (vgl. Dingeldey, Schröder \& Kathmann, 2015).

Ist gewerkschaftliches Handeln auf Inklusion gerichtet, sollten Beschäftigte mit geringer individueller Verhandlungsmacht davon profitieren. Eine Inklusionsstrategie müsste erstens zu einer Nivellierung der Lohnungleichheit innerhalb der Arbeiterschaft führen, da sich die Lohnsetzung an mittleren Tarifstandards orientiert. Folglich müsste ein hoher Organisationsgrad gerade den Frauenberufen zugutekommen, deren Löhne am unteren Ende der Lohnverteilung verortet sind. Zweitens kann argumentiert werden, dass Gewerkschaften vor allem Frauen schützen, da sie aufgrund vorherrschender Geschlechterstereotype in individuellen Lohnverhandlungen relativ stark benachteiligt sind (Stuhlmacher \& Walters, 1999; Mazei et al., 2015). Als typisch maskulin geltende Eigenschaften, wie Durchsetzungsstärke, werden im Vergleich zu Eigenschaften, die als typisch feminin gelten, auf dem Arbeitsmarkt als wertvoller beurteilt (Kray \& Thompson, 2005). Solche Geschlechterstereotype, die einer gleichen Entlohnung von Frauen und Männern entgegenstehen, sind in Gehaltsverhandlungen sowohl in der Wahrnehmung der Arbeitgeber als auch in der eigenen Wahrnehmung der Arbeitnehmer wiederzufinden (Wieber \& Holst, 2015). In kollektiven Lohnverhandlungen sollte sich dieser Nachteil, dem Frauen bei einer individuellen Aushandlung ausgesetzt sind, nivellieren, der GPG sich entsprechend schließen.

Zielt gewerkschaftliches Handeln auf Schließung ab, würde sich trotz oder vielmehr aufgrund eines hohen NOG eine relative Benachteiligung von Frauen und Frauenberufen ergeben, da das sekundäre Machtgefälle innerhalb der Arbeitnehmerschaft (Offe \& Hinrichs, 1977) in den Vordergrund tritt. So lässt sich historisch nachweisen, dass geschlechtsspezifische Lohnunterschiede auch in der männlich dominierten Gewerkschaftsbewegung legitimiert sind (Gottschall \& Schröder, 2013) bzw. das primäre Ziel der gewerkschaftlichen Arbeit nicht der Ausgleich von Ungleichheiten zwischen den Geschlechtern ist (Gärtner et al., 2014). Es kann argumentiert werden, dass Männerberufe aufgrund ihrer längeren Tradition gewerkschaftlicher Organisation und der entsprechend höheren Organisationsmacht besser entlohnt werden. In der Literatur zur Geschlechtersegregation wird die ungleiche Bezahlung in Männer- und Frauenberufen mit einer gesellschaftlichen Abwertung von Frauentätigkeiten begründet (vgl. Achatz, Gartner \& Glück, 2005). So gilt die „Affinität der Tätigkeiten zur Reproduktionsarbeit“, die ehemals unentgeltlich in den Familien geleistet wurde, als ,zentrales Unterscheidungskriterium“ zu Männertätigkeiten (Friedmann \& Pfau, 1985: 166f.). Dieser 
Mechanismus der horizontalen Abgrenzung und gleichzeitigen Abwertung von Berufen wird im Rahmen der Schließungstheorie auch als „Demarkation“ bezeichnet (Witz, 1990).

In der Literatur nicht immer klar unterschieden wird, ob Frauen im Erwerbssystem insgesamt von derartigen Abwertungsprozessen betroffen sind oder ob dies lediglich die historisch institutionalisierten Frauenberufe betrifft. Folgt man Ackers $(1991,1992)$ Annahme eines umfassenden „gendering“, kommt es in den sozialen Praktiken innerhalb von betrieblichen Organisationen sowie sozialen Institutionen, wie dem Beruf, zusammen mit einer symbolischen Differenzierung von Frauen und Männern immer auch zu einer hierarchischen Unterordnung von Frauen. Es finden sich Beispiele dafür, dass der Lohnsetzungsprozess trotz hohem gewerkschaftlichem Organisationsgrad zu einer Ausweitung des GPG innerhalb von Betrieben führt (Acker, 1991). Beispiel einer sozialen Praktik wäre die mittelbare Diskriminierung von Frauen durch Arbeitsbewertungssysteme und die Einordnung in untere Tariflohngruppen etwa aufgrund geringerer Berufserfahrung, an deren Etablierung die Gewerkschaften immer auch beteiligt sind (Carl \& Krehnke, 2004).

Zugleich kann gemutmaßt werden, dass Berufe historisch eine spezifisch vergeschlechtlichte institutionelle Ausprägung erfahren. So stellt die Literatur zum „comparable worth“ fest, dass Frauenberufe gegenüber Männerberufen trotz vergleichbarer Tätigkeiten historisch abgewertet werden (Treiman \& Hartmann, 1981; England, 2007). Frauen müssen innerhalb dieser Berufstypen nicht noch zusätzlich benachteiligt sein, sodass beide Geschlechter von einer geringen Organisationsmacht und entsprechend niedrigen Löhnen betroffen wären.

Demgegenüber geht die so genannte „Tokenism“-These Kanters (1977) davon aus, dass Geschlechterunterschiede sehr wohl zu beobachten sind, nur jeweils innerhalb von geschlechtsuntypischen Berufen. Bei einer sozialen Gruppe, etwa einer gewerkschaftlich organisierten Berufsgruppe, die allein hinsichtlich eines askriptiven Merkmals, wie des Geschlechts, ungleich verteilt ist, so die Annahme, tritt gerade dieses Merkmal besonders hervor. Die wenigen Zugehörigen zur Minderheitengruppe erhalten den Status eines stereotyp generalisierten Symbols („,token“) für das askriptive Merkmal selbst, das so in der Wahrnehmung und im Unterschied zur dominanten Gruppe stark hervortritt. Die Minderheitengruppe wird als mögliche Bedrohung sozial isoliert und steht zugleich unter einem besonderen Aufmerksamkeitsdruck. Eine typische Strategie der ,tokens` ist es, diesen Druck durch ,soziale Unsichtbarkeit‘ zu reduzieren und die eigenen Interessen in den Hintergrund zu stellen. Demzufolge sollte der Einfluss des NOG auf den Lohn für Frauen in Männerberufen geringer sein. Umgekehrt sollte dies jedoch ebenso für Männer in Frauenberufen gelten.

Einige Argumente sprechen indes gemäß der Gendering-These Ackers (s.o.) dafür, dass Frauen sowohl in Frauen- als auch Männerberufen benachteiligt werden (Budig, 2002). So merkt Yoder (1991) an, dass weniger die Strategien der Tokens als Diskriminierungsprozesse durch die dominante Gruppe selbst wirksam sind. Und diese erweisen sich als patriarchalisch geprägt: Frauen stoßen in Männerberufen auf ihrem Aufstiegsweg an eine „gläserne Decke“, während Männer in Frauenberufen den „gläsernen Fahrstuhl“ benutzen können (Williams, 1992). Diese Annahme deckt sich mit dem Forschungsergebnis, dass Männer auch in Frauenberufen besser bezahlt werden (Kunze, 2005; Busch, 2013).

Weniger im Sinne sozialer Schließung als eher auf Basis der Präferenzen von Frauen lässt sich schließlich eine Vereinbarkeitsthese formulieren. Demgemäß ist zu vermuten, dass Frauen insbesondere in Frauenberufen mit einer entsprechend „weiblichen“ Organisationsmacht eher gute Bedingungen der Vereinbarkeit von Beruf und Familie aushandeln - auf Kosten der Löhne (Heinze \& Wolf, 2010). 
Empirische Studien zu den rezipierten Theorien sind rar. Im internationalen Vergleich ausführlich untersucht wurde lediglich die Frage, inwiefern sich verschiedene Dimensionen kollektiver Interessenvertretung auf die Löhne insgesamt auswirken. Das Gros der Befunde deutet auf eine Nivellierung der Lohnungleichheit durch Gewerkschaften hin (für einen Überblick siehe Bryson, 2007). Da sich die Systeme industrieller Beziehungen zwischen einzelnen Ländern zum Teil fundamental unterscheiden, können internationale Studien nur grobe Hinweise für den deutschen Fall liefern. ${ }^{5}$ Dies gilt vor allem auch für Aussagen zum Einfluss der Gewerkschaften auf den GPG. Hier kommt noch die unterschiedliche Beschäftigungsstruktur von Frauen und Männern hinzu (zu internationaler Literatur und den deutschen Besonderheiten siehe Schäfer \& Gottschall, 2015). Blau und Kahn (2003) stellen in einer Untersuchung von 14 Ländern eine nivellierende Wirkung des Tarifbindungsgrades auf den GPG fest. In dieselbe Richtung weisen die Ergebnisse einer Studie mit 26 EU-Ländern (Christofides et al., 2013). Länder mit gewerkschaftlich unregulierten Arbeitsmärkten weisen einen höheren GPG auf. Schäfer und Gottschall (2015) hingegen finden im Vergleich von Vollzeitbeschäftigten in 25 EU-Ländern Hinweise darauf, dass Frauen in Ländern mit hohem Zentralisierungsgrad der Lohnverhandlungen gegenüber Männern im Lohn eher benachteiligt werden. Sie interpretieren dies als Dominanz einer „männlichen Lohnverhandlungskultur" und gehen somit implizit von einer Schließungsthese aus. Blickt man in einzelne Länder, so zeigen sich zum Teil gegenläufige Befunde. In Irland etwa profitieren Frauen insgesamt von zentralen Lohnverhandlungen, während dies bei den Männern nur für Teilzeiterwerbstätige der Fall ist (McGuinnes et al., 2011). Even und Macpherson (1993) machen den abnehmenden NOG unter Männern verantwortlich für das Sinken des GPG in den USA. Die meisten Studien finden für die USA zudem einen geringeren GPG in gewerkschaftlich geschützten Arbeitsverhältnissen (Elvira \& Saporta, 2001; Cho \& Cho, 2011).

Der Einfluss von Gewerkschaften auf den GPG wurde für die BRD ungleich seltener analysiert. Es findet sich Evidenz dafür, dass die Tarifbindung mit der allgemeinen Lohnungleichheit auch den GPG reduziert (Heinze \& Wolf, 2010; Antonczyk, Fitzenberger, \& Sommerfeld, 2010; Cho \& Cho, 2011). Dieser Befund deutet auf eine Inklusionsstrategie der Gewerkschaften in Bezug auf Frauen hin. Zugleich finden sich Hinweise darauf, dass Frauen auch dann, wenn ihre Löhne tarifvertraglich geregelt sind, wesentlich seltener als Männer an tariflichen Facharbeiterlöhnen orientierten, so genannte Ernährerlöhne erzielen (Schröder \& Schäfer, 2013). ${ }^{6}$ Dieser Befund steht allerdings nicht im Widerspruch zur Nivellierung am unteren Ende der Lohnverteilung, da sich Ernährerlöhne am oberen Ende befinden. Der Zusammenhang zwischen der Machtressource des NOG und der Lohnhöhe wurde in Deutschland bislang hauptsächlich für männliche Vollzeitbeschäftigte untersucht. Es zeigt sich, dass ein hoher NOG die Lohnungleichheit reduziert, indem die Löhne vor allem am oberen Ende (Fitzenberger, Kohn \& Lembcke, 2013) der Lohnverteilung gesenkt und am unteren Ende im Sinne eines Mindestlohnes angehoben werden (Fitzenberger \& Kohn, 2005). Dies allerdings gilt nur für tariflich gebundene Arbeitnehmer.

5 Unterschiede bestehen vor allem im Zusammenhang des Zentralisierungsgrads kollektiver Lohnverhandlungen, von NOG und Tarifbindung sowie in gesetzlichen Regelungen, wie etwa der negativen Koalitionsfreiheit, die eine Diskriminierung nicht-gewerkschaftlich organisierter Beschäftigter in Betrieben verhindern soll.

6 Der Ernährerlohn ist hierbei definiert als der Median des Bruttolohns der Männer in einer abhängigen, sozialversicherungspflichtigen Vollzeitbeschäftigung mit einer berufsfachlichen Ausbildung oder einem Fachhochschulabschluss und mindestens zweijähriger Betriebszugehörigkeit, die in den klassischen Lohnführerbranchen der Metall- oder Automobilindustrie tätig sind. 
Mit Blick auf einen Vergleich der Geschlechtereffekte des NOG zwischen segregierten Branchen und Berufen finden sich nur vereinzelt internationale Studien. Elvira und Saporta (2001) finden branchenspezifische Effekte für den Industriesektor in den USA: In den Branchen mit dem höchsten Frauenanteil zeigt sich ein gegenläufiger Effekt des innerbetrieblichen NOG zum allgemeinen, den GPG reduzierenden Trend. Hinter dem Effekt wird eine schwache Position weiblich dominierter gegenüber männlich dominierten Gewerkschaften in denselben Betrieben vermutet. Die institutionelle Struktur in Deutschland macht derartige Konkurrenzsituationen von Gewerkschaften innerhalb von Betrieben allerdings unwahrscheinlich. Gemäß der Comparable-Worth-These wären Frauen und Männer einerseits gleichermaßen von einer schwachen Lohnverhandlungsposition weiblich dominierter Gewerkschaften gegenüber den Arbeitgebern betroffen. Andererseits ist der obige Befund kompatibel mit der Vereinbarkeitsthese, wonach Vereinbarkeitsbedingungen auf Kosten der Löhne lediglich die weiblichen Beschäftigten beträfen. Diese Vermutung bestätigend, stellen Wunnava und Ewing (2000) für die USA einen Zusammenhang von betrieblichen Elternschaftsregelungen und dem NOG von Frauen fest.

Inwiefern die für Deutschland festgestellte Nivellierung der Lohnungleichheit durch den NOG auch den Frauen zugutekommt und in welchen Berufstypen - Männer-, Frauen- oder Mischberufen - Gewerkschaften zur Inklusion von Frauen und somit zur Reduzierung des GPG beitragen oder ob von sozialen Schließungsstrategien auszugehen ist, welche den GPG erhöhen, wird im Folgenden empirisch untersucht.

Im Hinblick auf Theorie und Empirie können unterschiedliche Hypothesen generiert werden. Die Empirie deutet darauf hin, dass Gewerkschaften grundsätzlich im Sinne einer Inklusionsstrategie handeln. Hieraus folgt die Hypothese:

H1) Ein hoher NOG verringert den GPG.

Folgt man der Hypothese H1, ist mit Blick auf Unterschiede im Zusammenhang von NOG und GPG zwischen den Berufstypen nicht von einer generellen Benachteiligung von Frauen auszugehen. Darüber hinaus lässt sich aus der Empirie keine eindeutige Hypothese ableiten. Wir formulieren daher im Folgenden drei konkurrierende Hypothesen. Gemäß der Comparable-Worth-These sollten die Gewerkschaften in Frauenberufen eine geringe Verhandlungsmacht aufweisen, das niedrigere Lohnniveau gilt indes für beide Geschlechter gleichermaßen. Entsprechend kann vermutet werden, dass

H2) der NOG in Frauenberufen keinen Effekt auf den GPG hat.

Folgt man der Tokenism-These, bei der die jeweilige Minderheit in einer Gruppe benachteiligt wird, sollte

H3) der NOG in Männerberufen den GPG erhöhen und in Frauenberufen verringern. Gemäß der Vereinbarkeitsthese würde

H4) der NOG in Frauenberufen den GPG vergrößern, da sich weibliche Gewerkschaftsmitglieder zu Ungunsten der Löhne für familienfreundliche Arbeitsbedingungen einsetzen. Zu der Wirkung des NOG auf den GPG in den Mischberufen kann auf Grundlage der Theorien keine gesonderte Hypothese abgeleitet werden. Die Untersuchung dieses Berufstyps hat entsprechend einen eher explorativen Charakter bzw. kann sie als Referenzgruppe zu den Männer- und Frauenberufen gesehen werden. 


\section{Daten und Methode}

\section{Daten und Stichprobe}

Als Datensatz wird das Sozio-oekonomische Panel (SOEP) genutzt. Das SOEP ist eine seit 1984 existierende, jährliche, repräsentative Wiederholungsbefragung von Privathaushalten in Deutschland. Aus den Daten wird eine Substichprobe für die Jahre 2001 bis 2012 generiert, in der sich nur abhängig Beschäftigte im Alter zwischen 25 und 60 Jahren befinden. Der NOG wird operationalisiert, indem die individuelle Gewerkschaftsmitgliedschaft nach Branchen-Berufs-Zellen, das heißt nach Berufen in Branchen, aggregiert wird. Beispielsweise werden Bürokaufleute im Baugewerbe einer anderen Zelle zugeordnet als Bürokaufleute in der öffentlichen Verwaltung. Dies trägt einerseits dem Tatbestand Rechnung, dass Tarifverhandlungen zumeist auf Branchenebene stattfinden und berücksichtigt zudem die berufliche Geschlechtersegregation in diesen Branchen (vgl. Kaufman, 2002; Weeden \& Sørensen, 2004; Fitzenberger, Kohn \& Lembcke, 2013). Branchen-Berufs-Zellen, die über alle Jahre eine Zellbesetzung von unter 30 Fällen aufweisen ${ }^{7}$, werden aus den Analysen entfernt. Des Weiteren schließen wir Personen aus, die fehlende Werte in einer der im Modell verwendeten Variablen aufweisen. Der Anteil der fehlenden Werte in der Stichprobe liegt bei 11,7\% und ist hauptsächlich auf die Variable „Branchen“ zurückzuführen (4,5\% Missings). Bei den anderen Variablen rangiert der Anteil an fehlenden Werten zwischen 0 und 3,1\%. Entsprechend dieser Selektion ergibt sich für den kompletten Untersuchungszeitraum eine (ungewichtete) Fallzahl von 80.718 in 3.551 Branchen-Berufs-Zellen und 12 Jahren.

\section{Variablen}

Die abhängige Variable ist der Bruttostundenlohn, der aus dem Bruttomonatslohn dividiert durch die tatsächlich im Monat geleisteten Arbeitsstunden generiert wird. Aufgrund ihrer schiefen Verteilung und den daraus resultierenden Problemen bei der Anwendung von Regressionsverfahren wird die abhängige Variable logarithmiert. Die Regressionskoeffizienten können entsprechend näherungsweise als prozentuale Veränderungen des Lohns interpretiert werden.

Die zentralen erklärenden Variablen sind das Geschlecht (Kodierung: Frau =1, Mann = 0 ) und der Nettoorganisationsgrad der Gewerkschaften nach Branchen-Berufs-Zellen. Hier wird auf die Berufsklassifikation des statistischen Bundesamtes auf 2-Steller-Ebene zurückgegriffen und die Branchen sind nach den 21 Abschnitten gemäß NACE Rev. 2 operationalisiert. Die Gewerkschaftsmitgliedschaft wurde im SOEP nicht jedes Jahr, sondern im Analysezeitraum nur in den Jahren 2001, 2003, 2007 und 2011 erhoben. Aufgrund der niedrigen Fallzahlen pro Jahr in einigen Branchen-Berufs-Zellen wird ein Gesamt-NOG für den kompletten Analysezeitraum berechnet. Konkret wird die Anzahl der Mitglieder durch die Anzahl der Beschäftigten in den jeweiligen Branchen-Berufs-Zellen dividiert. Da das erste Quartil des NOG bei 10,7\% und das dritte Quartil bei 26\% liegt, wurde der NOG zur besseren Interpretation der Ergebnisse nicht in Prozent, sondern in Zehntelprozent umgerechnet. Die Koeffizienten des NOG stellen somit dar, um wieviel Prozent sich der Stundenlohn ändert, wenn sich der NOG um 10\% ändert. In den Partialmodellen wird nach Frauen-, Misch- und Männerberufen unterschieden. Der Schwellenwert wird hier jeweils auf 70 Prozent gesetzt, d.h. 
dass ein Frauenberuf einen Frauenanteil von 70 oder mehr Prozent aufweist und vice versa für die Männerberufe (vgl. Busch, 2013). Die Mischberufe ergeben sich aus der Restmenge.

Um den Effekt des NOG auf den GPG korrekt zu schätzen, bedarf es der Kontrolle relevanter Merkmale. Wir orientieren uns hierbei an den in der Literatur zum GPG rezipierten aktuellen Erweiterungen der humankapitaltheoretischen, so genannten Mincer-Lohngleichungen (Mincer, 1974). Diese Erweiterungen berücksichtigen, neben Bildung, allgemeiner Berufserfahrung und Betriebszugehörigkeit, auch die spezifischen Besonderheiten des familiären Lebenslaufs, der vor allem die weiblichen Erwerbsbiografien prägt (Beblo \& Wolf, 2003; Busch, 2013). So werden etwa Familienstand, Kinderzahl, aktueller Arbeitszeitumfang und vor allem auch die Berufserfahrung in Vollzeitbeschäftigung einbezogen. Obgleich betriebliche Merkmale auf der Arbeitskraftnachfrageseite (Schröder, Struck \& Wlodarski, 2008) sowie der Produktmarktwettbewerb (Hirsch, Oberfichtner \& Schnabel, 2014) ebenfalls den Lohn beeinflussen, werden in den Modellen datenbedingt nur die Betriebsgröße und der öffentliche Dienst berücksichtigt. Ein Überblick über die Verteilung der Variablen in den Untersuchungsgruppen befindet sich im Anhang (Tab. 1.A). ${ }^{8}$

Tab. 1: Operationalisierung der Kontrollvariablen

\begin{tabular}{|l|l|}
\hline Variable & Operationalisierung \\
\hline Zeit & Jahre als Dummy-Variablen \\
\hline Alter & in Jahren \\
\hline Familienstand & $\begin{array}{l}1=\text { verheiratet; } 2 \text { = nicht-eheliche Lebensgemeinschaft; } 3=\text { Single und Personen, die nicht mit } \\
\text { dem Partner zusammen leben }\end{array}$ \\
\hline Kinderzahl & $0=$ keine Kinder; 1 = ein Kind; 2 = zwei Kinder; 3 = drei und mehr Kinder \\
\hline Bildung & ISCED 1-2 = niedrige Bildung; ISCED 3-4 = mittlere Bildung; ISCED 5-6 = hohe Bildung \\
\hline Berufserfahrung & Dauer der Vollzeitberufserfahrung in Monaten \\
\hline Berufsprestige & Index of Occupational Prestige Scala (SIOPS) (auch Treiman-Index) \\
\hline Arbeitsumfang & $1=$ vollzeiterwerbstätig; 2 = teilzeitbeschäftigt, 3 = unregelmäßig/geringfügig beschäftigt \\
\hline Überstunden & Überstunden pro Woche \\
\hline Betriebszugehörigkeit & Dauer der Betriebszugehörigkeit in Jahren \\
\hline Unternehmensgröße & $\begin{array}{l}1=\text { bis unter 20 Beschäftigte; 2 = 20 bis unter 200 Beschäftigte; 3 = 200 bis unter 2000 Beschäf- } \\
\text { tigte; 4 = 2000 und mehr Beschäftigte }\end{array}$ \\
\hline Öffentlicher Dienst & $0=$ Nein; 1 = Ja \\
\hline Region & $0=$ Westdeutschland; 1 = Ostdeutschland \\
\hline
\end{tabular}

Methode

Neben den inhaltlichen Vorzügen besteht der methodische Vorteil des Sozio-oekonomischen Panels darin, dass die Paneldatenstruktur genutzt werden konnte, um eine hinreichende Zellenbesetzung in der zentralen unabhängigen Variable, dem NOG nach Branchen-Berufs-Zellen, zu erreichen. Der NOG ist hierzu über verschiedene Messzeitpunkte gepoolt worden und

8 Auf die üblicherweise quadrierten Terme für Alter, Berufserfahrung und Betriebszugehörigkeit zur Kontrolle nichtlinearer Effekte konnte in den Gesamtmodellen verzichtet werden. Einzig der quadrierte Alterseffekt ist schwach signifikant negativ. Um das Modell sparsam zu halten, wurde diese Variable ausgelassen. Die Modellkoeffizienten ändern sich hierdurch nur marginal. Die Konstruktion der Dummyvariablen für den Unternehmensgrößeneffekt bildet in etwa eine logarithmische Transformation ab. 
somit zeitkonstant. Längsschnittanalysen, wie Fixed-Effects- oder Wachstumskurven-Modelle, sind aus diesem Grunde nicht möglich (Giesselmann \& Windzio, 2012: 69ff.). Aufgrund der hierarchischen Datenstruktur - Messzeitpunkte sind in Personen und Personen sind in Branchen-Berufs-Zellen genestet - kann auch eine einfache lineare Regression nicht zur Anwendung kommen. Stattdessen wird ein Mehrebenen-Random-Effects-Modell mit drei Ebenen geschätzt (Hox, 2002; Rabe-Hesketh \& Skrondal, 2012). Das lineare Mehrebenenmodell kann konzeptuell als hierarchisches System von Regressionsgleichungen aufgefasst werden. Im Folgenden werden die Gleichungen ohne erklärende Variablen für die drei Ebenen (1) Messzeitpunkte, (2) Personen und (3) Branchen-Berufs-Zellen dargestellt.

$$
\begin{array}{llll}
\text { (1) } & Y_{i j k}=\beta_{0 j k}+\varepsilon_{i j k} & \text { mit } & \operatorname{Var}\left(\varepsilon_{i j k}\right)=\sigma^{2} ; \varepsilon_{i j k} \sim N\left(0, \sigma^{2}\right) \\
\text { (2) } & \beta_{0 j k}=\gamma_{00 k}+u_{0 j k} & \text { mit } & \operatorname{Var}\left(u_{0 j k}\right)=\tau_{0}^{2} ; u_{0 j k} \sim N\left(0, \tau_{0}^{2}\right) \\
\text { (3) } & \gamma_{00 k}=\delta_{000}+v_{00 k} & \text { mit } & \operatorname{Var}\left(v_{00 k}\right)=\emptyset_{0}^{2} ; v_{00 k} \sim N\left(0, \emptyset_{0}^{2}\right)
\end{array}
$$

Der Index i steht für den Messzeitpunkt, $i$ steht für das Individuum und $k$ für die verschiedenen Branchen-Berufs-Zellen. Abweichend von einer OLS-Regression beinhaltet die Gleichung drei anstatt eines einzelnen Fehlerterms. Werden die Formeln ineinander eingesetzt und durch erklärende Variablen ergänzt, kann der logarithmierte Bruttostundenlohn $\left(Y_{i j k}\right)$ durch folgende Gleichung dargestellt und konsistent geschätzt werden:

$$
Y_{i j k}=\delta_{000}+\beta_{1 j k}+\ldots+\beta_{n j k}+\varepsilon_{i j k}+u_{0 j k}+v_{00 k}
$$

\section{Ergebnisse}

\section{Deskriptive Befunde}

Die folgende Tabelle 2 zeigt Differenzen zwischen Männer-, Frauen- und Mischberufen im Hinblick auf Lohn, NOG und einige weitere Beschäftigungsmerkmale in diesen Berufstypen. Frauen- und Männerberufe unterscheiden sich stark in ihrem NOG, der innerhalb der Männerberufe fast doppelt so hoch ist. Auch die geschlechtsspezifische Zusammensetzung differiert. In den Frauenberufen sind gut zwei Drittel der Gewerkschaftsmitglieder Frauen', während es bei den Männerberufen nicht mal jede Zehnte ist. Heinze und Wolf (2010) konnten im Hinblick auf diese Dimension zeigen, dass die Tarifbindung in Branchen mit einem hohen Anteil an weiblichen Gewerkschaftsmitgliedern mit einem höheren GPG einhergeht. Sie folgern hieraus, dass sich Frauen in Gewerkschaften primär für die Vereinbarkeit von Familie und Beruf und weniger für Lohnerhöhungen einsetzen. Eine Teilzeiterwerbstätigenquote von fast $50 \%$ in den Frauenberufen kann als Hinweis dienen, dass diese Annahme zutreffen könnte. In den Frauenberufen werden im Schnitt überdies weniger Überstunden geleistet (1,8 pro Woche). Die durchschnittliche Anzahl an Überstunden ist allerdings in den Misch- und nicht in den Männerberufen am höchsten und liegt dort bei 2,8 Stunden pro Woche. Vergleichsweise ähnlich sind sich Frauen- und Männerberufe im Berufsprestige und liegen relativ zu den Mischberufen auch im Akademikeranteil näher beieinander. In den Mischberufen liegt der Akademikeranteil bei über 50\%, was mit einem überdurchschnittlichen Berufsprestige einhergeht. Die Mischberufe haben ebenso den höchsten Median-Lohn,

9 Tabelle 2 zeigt darüber hinaus, dass der NOG der Frauen in allen Berufstypen z.T. weit unter dem der Männer liegt. Gewerkschaften scheinen von Frauen seltener als Institution betrachtet zu werden, innerhalb der sie ihre Rechte vertreten können. 
die höchste Lohnspreizung und den höchsten GPG. Letzterer ist bei den Männerberufen am geringsten. Die Ergebnisse in Tabelle 2 bestätigen das bisherige Forschungsergebnis, dass Männer auch in Frauenberufen besser bezahlt werden (Kunze, 2005; Busch, 2013). Hier liegt der (unbereinigte) GPG bei knapp 20\%.

Die Masse der Berufe sind auf sieben Wirtschaftszweige der 21 Abschnitte der NACE Rev. 2 verteilt. Unter den Männerberufen sind knapp die Hälfte $(47,1 \%)$ im verarbeitenden Gewerbe oder der Herstellung von Waren tätig. Hier ist auch ein Viertel der Mischberufe verortet. Die zweitstärkste Branche der Männerberufe ist das Baugewerbe (13,2\%). Die insgesamt am zweitstärksten besetzte Branche ist der Handel sowie die KFZ-Reparatur. Hier sind $14,8 \%$ der Misch- und 15,3\% der Frauenberufe angesiedelt. Die drittgrößte Branche unter den Männer- und Frauenberufen ist mit jeweils gut 11\% der Sektor „oeffentliche Verwaltung, Verteidigung, Soziales“. Die Branche Erziehung und Unterricht ist die am drittstärksten besetzte Branche unter den Mischberufen (14,3\%). Jeder zehnte Arbeitnehmer in den Mischberufen ist im Bereich „Erbringung von Finanz- und Versicherungsdienstleistungen“ tätig. Fast ein Drittel der Frauenberufe ist im Zweig „Gesundheits- und Sozialwesen“ verortet.

Tab. 2: Merkmale in Frauen-, Misch- und Männerberufen (wo nicht anders angegeben, in \%)

\begin{tabular}{|c|c|c|c|c|}
\hline & Männerberufe & Mischberufe & Frauenberufe & Gesamt \\
\hline Median-Bruttostundenlohn in EUR & 13,9 (Std.abw.: 7,7) & 16,8 (Std.abw.: 11,4) & 11,6 (Std.abw.: 7,5) & 14,0 (Std.abw.: 9,5) \\
\hline GPG & 15,5 & 24,7 & 19,8 & 22,9 \\
\hline$\varnothing$ NOG (davon Frauen) & $27,8(7,8)$ & $16,6(40,6)$ & $15,5(68,8)$ & $18,9(34,9)$ \\
\hline$\varnothing$ NOG Männer & 28,8 & 20,2 & 20,2 & 24,4 \\
\hline$\varnothing$ NOG Frauen & 17,4 & 14,2 & 12,2 & 13,3 \\
\hline Anteil Vollzeiterwerbstätige & 91,8 & 78,6 & 53,2 & 73,2 \\
\hline$\varnothing$ Überstunden pro Woche & 2,4 & 2,8 & 1,8 & 2,3 \\
\hline$\varnothing$ SIOPS-Prestige & 40,1 & 50,1 & 40,8 & 44,3 \\
\hline Akademikeranteil & 27,2 & 52,3 & 21,7 & 34,8 \\
\hline Branchen & $\begin{array}{l}\text { - } \quad \text { Verarbeitendes } \\
\text { Gewerbe/Herstellung } \\
\text { von Waren }(47,1 \%) \\
\text { - } \quad \text { Baugewerbe }(13,2 \%) \\
\text { - oeff. Verwaltung, } \\
\text { Verteidigung, } \\
\text { Soziales }(11,5 \%)\end{array}$ & $\begin{array}{ll}\text { - } & \text { Verarbeitendes } \\
& \text { Gewerbe/Herstellung } \\
& \text { von Waren }(25,0 \%) \\
\text { - } & \text { Handel; KFZ } \\
& \text { Reparatur }(14,8 \%) \\
\text { - } & \text { Erziehung und } \\
& \text { Unterricht }(14,3 \%) \\
\text { - } & \text { Erbringung von } \\
& \text { Finanz- und } \\
& \text { Versicherungsdienst- } \\
\text { leistungen }(11 \%)\end{array}$ & $\begin{array}{ll}\text { - } & \text { Gesundheits- und } \\
& \text { Sozialwesen } \\
(32,2 \%) \\
\text { - } & \text { Handel; KFZ } \\
& \text { Reparatur }(15,3 \%) \\
\text { - } & \text { oeff. Verwaltung, } \\
\text { Verteidigung, } \\
\text { Soziales }(11,7 \%)\end{array}$ & $\begin{array}{ll}\text { - } & \text { Verarbeitendes } \\
& \text { Gewerbe/Herstellung von } \\
& \text { Waren }(25,5 \%) \\
\text { - } & \text { Gesundheits- und } \\
& \text { Sozialwesen }(13,0 \%) \\
\text { - } & \text { Handel; KFZ Reparatur } \\
& (12,9 \%) \\
\text { - } & \text { oeff. Verwaltung, } \\
\text { Verteidigung, Soziales } \\
(10,2 \%)\end{array}$ \\
\hline
\end{tabular}

Quelle: SOEPv30 (2001-2012), eigene, gewichtete Berechnungen

\section{Ergebnisse der Mehrebenenmodelle}

Tabelle 3 zeigt die Ergebnisse der Regressionsanalysen des logarithmierten Stundenlohnes. Berücksichtigt werden in der Tabelle nur die Koeffizienten der Variablen von zentralem Interesse: des Geschlechts, des Berufstyps, des NOG nach Branchen-Berufs-Zellen sowie der Interaktionseffekte von Geschlecht und NOG. ${ }^{10}$ Dargestellt werden sechs Modelle. Die Modelle M1-M3 beziehen sich auf die Gesamtstichprobe aller Berufe. Hier werden die theoretisch bedeutsamen Variablen schrittweise hinzugefügt. In den Modellen M4-M6 wird die Stichprobe nach Berufstypen differenziert.

Das erste Modell für alle Berufe (M1) enthält, neben den Kontrollvariablen, lediglich den Effekt des individuellen Geschlechts sowie die Unterscheidung nach geschlechtstypischen Berufen. Es ergibt sich ein bereinigter GPG von 12,4\%. Fast die Hälfte des unbereinigten

10 Die zudem berücksichtigten Kontrollvariablen sind unter der Tabelle aufgeführt, die Ergebnisse der Gesamtmodelle finden sich in den Tabellen 2.A und 3.A im Anhang. 
GPG kann somit durch die Variablen des Modells erklärt werden. Wie bereits deskriptiv festgestellt, müssen Beschäftigte in Frauenberufen Lohnabschläge gegenüber Männerberufen hinnehmen (1,6\%), während in Mischberufen relativ höhere Löhne erzielt werden (7,2\%). Im zweiten Modell (M2) wird zusätzlich der NOG nach Branchen-Berufs-Zellen berücksichtigt: Mit einer Zunahme des NOG um 10\% sind um knapp 0,7\% höhere Löhne verbunden. Es zeigt sich, dass dieser Effekt den zuvor noch festgestellten Lohnunterschied zwischen Männer- und Frauenberufen erklärt. Die Löhne in den Männerberufen sind m.a.W. unter anderem deshalb höher, weil Frauenberufe einen geringeren NOG aufweisen.

Die nun folgenden Modelle enthalten für alle Berufe (M3) sowie die drei Berufstypen (M4-M6) jeweils die Interaktionseffekte des NOG mit dem Geschlecht und zeigen somit auf, inwieweit sich der Lohneffekt des NOG nach Geschlecht unterscheidet. Um die Haupt- und Interaktionseffekte besser interpretieren zu können, sind in Abbildung 1 für alle Interaktionsmodelle die vorhergesagten Löhne für unterschiedliche Nettoorganisationsgrade dargestellt. ${ }^{11}$ Im dritten Modell (M3) für alle Berufe wird deutlich, dass eine Zunahme des NOG (um 10\%) mit einem Lohngewinn ausschließlich für Frauen (von 1,4\%) verbunden ist. Dies führt in der Konsequenz zu einer Verringerung des GPG.

Wirft man einen Blick auf die Berufstypen, so werden Unterschiede deutlich. Erstens können Männer in Männerberufen (M4), anders als im Modell für alle Berufe, in gleichem Maße wie Frauen Lohngewinne erzielen, wenn sie gewerkschaftlich organisiert sind. Mit einer Zunahme des NOG von 10\% steigen die Löhne der Männer um 1,1\%, der Anstieg der Frauenlöhne ist nur unwesentlich größer. Der bereinigte GPG verändert sich mit steigendem NOG nur marginal und verharrt bei etwa 10\%. Zweitens können in Frauenberufen (M4) ausschließlich Frauen durch einen höheren NOG (bei einem Anstieg um 10\%) auch höhere Löhne (um 2,4\%) realisieren. ${ }^{12}$ Der bereinigte GPG von 14,7\% würde sich bei einem NOG von $40 \%$ schließen, d.h. die Männerlöhne würden sich nicht mehr signifikant von den Frauenlöhnen unterscheiden (Abb. 1). Drittens weisen Mischberufe (M6) den höchsten bereinigten GPG auf (17\%). Auch hier schließt sich der GPG mit zunehmendem NOG - einerseits, wie in den Frauenberufen, dadurch, dass Frauen höhere Löhne realisieren (um 1,9\%). Andererseits müssen Männer hier sogar Lohnverluste in Kauf nehmen (-1,9\%). Bei einem, allerdings unrealistisch hohen, NOG von 50\% wäre der GPG nahezu vollständig geschlossen. Insgesamt findet sich also sowohl in den Frauenberufen als auch den Mischberufen ein die Geschlechterungleichheit nivellierender Effekt des NOG. Dieser Effekt zeigt sich nicht in den Männerberufen, wo sich allerdings bereits ein geringer durchschnittlicher GPG zeigt.

Interpretiert man diese Ergebnisse mit Blick auf die Hypothesen, ergibt sich folgendes Bild: Die Inklusionshypothese (H1) mit der Annahme, dass sich mit Erhöhung des NOG der GPG verringert, wird untermauert (M3). Im Hinblick auf die gewerkschaftliche Nutzung der Organisationsmacht lässt sich also zunächst eher eine Inklusions- als eine Schließungswirkung vermuten. Die nach Berufstypen differenzierten Analysen zeigen jedoch, dass sich die Machtressourcen und ihre Nutzung in Männer-, Frauen- und Mischberufen zu unterscheiden scheinen. Allerdings widersprechen die Befunde hierbei den Hypothesen zu den in unterschiedlichen Berufstypen wirksamen Mechanismen sozialer Schließung beziehungsweise der Vereinbarkeitsthese.

11 Dabei werden für alle Kontrollvariablen die Mittelwerte in der jeweiligen Stichprobe angenommen (vgl. Tab. 2 sowie Tab.1.A im Anhang).

12 Dieses Ergebnis zeigt sich auch, wenn man im Modell mit allen Berufen den Anteil an Frauen unter den Gewerkschaftsmitgliedern anstelle des allgemeinen NOG als Interaktionsterm mit der Variable „Frau“ einfügt. 
In Männerberufen (M4) steigen mit wachsendem NOG im Mittel die Löhne für beide Geschlechter. Ausschließlich in diesen männlich geprägten Berufen können Männer durch gewerkschaftliche Organisation Lohngewinne erzielen, allerdings nicht im Sinne sozialer Schließung auf Kosten der Frauenlöhne. Die Inklusionsthese trifft jedoch ebenso nur bedingt zu: Der GPG ist zwar insgesamt gering und gewerkschaftliche Organisation ist für Frauen von Vorteil, sie können sich aber hierdurch in ihrer relativen Position gegenüber den Männern nicht verbessern.

Die Ergebnisse in den Frauenberufen (M5) sind zwar einerseits kompatibel mit der „Tokenism"-These (H3), insofern die Machtressource des NOG ausschließlich den Frauen und nicht den Männern zugutekommt. Auf der anderen Seite trifft dies nicht für die Männer in Männerberufen zu, sodass die Tokenism-These insgesamt zurückgewiesen werden muss. Die Comparable-Worth-These trifft insofern zu, als das Lohnniveau in Frauenberufen zwar insgesamt geringer ist, in Bezug auf den Einfluss der Gewerkschaften muss die These indes ebenso verworfen werden: Die gewerkschaftliche Organisation kommt den Frauen sogar noch in stärkerem Maße zugute als in den Männerberufen. In diesem Sinne trifft auch die Vereinbarkeitsthese nicht zu, wonach die gewerkschaftlich organisierten Frauen eher Lohneinbußen zugunsten besserer Vereinbarkeitsbedingungen in Kauf nehmen. Im Ergebnis bestätigt sich in den Frauenberufen damit die Inklusionsthese.

Tab. 3: Drei-Ebenenmodelle logarithmierter Bruttostundenlohn

\begin{tabular}{|c|c|c|c|c|c|c|}
\hline & Alle Berufe (M1) & Alle Berufe (M2) & Alle Berufe (M3) & Männerberufe (M4) & Frauenberufe (M5) & Mischberufe (M6) \\
\hline Frau & $\begin{array}{c}-0,124^{* \star \star} \\
(0,004)\end{array}$ & $\begin{array}{c}-0.124^{\prime \prime \prime} \\
(0.004)\end{array}$ & $\begin{array}{c}-0.149^{\prime \prime \prime+*} \\
(0.006)\end{array}$ & $\begin{array}{c}-0.102^{\prime \prime} \\
(0.014)\end{array}$ & $\begin{array}{c}-0.147^{\prime \prime \prime} \\
(0.013)\end{array}$ & $\begin{array}{c}-0.170^{\prime *+1} \\
(0.009)\end{array}$ \\
\hline \multicolumn{7}{|c|}{ Berufstyp (Ref:: Männerberuf) } \\
\hline Mischberuf & $\begin{array}{c}0,071^{\star * *} \\
(0.007)\end{array}$ & $\begin{array}{l}0.077^{\prime \prime *} \\
(0.007)\end{array}$ & $\begin{array}{l}0.075^{w *} \\
(0.007)\end{array}$ & & & \\
\hline Frauenberuf & $\begin{array}{l}-0.016^{*} \\
(0.007)\end{array}$ & $\begin{array}{c}-0.009 \\
(0.008)\end{array}$ & $\begin{array}{c}-0.007 \\
(0.008)\end{array}$ & & & \\
\hline $\begin{array}{l}\text { NOG nach } \\
\text { Branchen-Berufs- } \\
\text { Zellen/10 }\end{array}$ & & $\begin{array}{c}0.007^{\star \star *} \\
(0.002)\end{array}$ & $\begin{array}{c}0.002 \\
(0.002)\end{array}$ & $\begin{array}{l}0.011^{1 *} \\
(0.002)\end{array}$ & $\begin{array}{c}0.002 \\
(0.006)\end{array}$ & $\begin{array}{c}-0.019^{\prime * * *} \\
(0.037)\end{array}$ \\
\hline $\begin{array}{l}\text { Frau*NOG nach } \\
\text { Branchen-Berufs- } \\
\text { Zellen/10 }\end{array}$ & & & $\begin{array}{l}0.014^{4 *} \\
(0.002)\end{array}$ & $\begin{array}{c}0.002 \\
(0.005)\end{array}$ & $\begin{array}{l}0.024^{* * *} \\
(0.007)\end{array}$ & $\begin{array}{l}0.019^{* * *} \\
(0.004)\end{array}$ \\
\hline Var (Jahre) & 0,000 & 0,000 & 0,0000 & 0,000 & 0,000 & 0,000 \\
\hline Var (Berufe) & 0,0115 & 0,0115 & 0,0115 & 0,0103 & 0,0060 & 0,0140 \\
\hline Residualvarianz & 0,1297 & 0,1300 & 0,1355 & 0,0969 & 0,1449 & 0,1345 \\
\hline $\mathrm{AIC} / \mathrm{BIC}$ & $67040 / 67384$ & $67029,71 / 67383,06$ & $70559,65 / 70922,33$ & $13102,79 / 13401,42$ & $24742,78 / 25045,90$ & $26946,06 / 27253,95$ \\
\hline $\mathrm{N}$ & 80.718 & 80.718 & 80.718 & 23.646 & 26.702 & 30.370 \\
\hline
\end{tabular}

Quelle: SOEPv30 (2001-2012), eigene Berechnungen

Anmerkungen: * $p<0.05,{ }^{* *} p<0.01,{ }^{* * *} p<0.001$, Standardfehler in Klammern

Weitere, nicht in der Tabelle aufgeführte Kontrollvariablen: Jahres-Dummies, Alter, Familienstand, Kinderzahl, Bildung, Berufsprestige nach Treiman, Dauer der Vollzeitberufserfahrung, Umfang der Erwerbstätigkeit, Dauer der Betriebszugehörigkeit, Überstunden, Öffentlicher Dienst, Unternehmensgröße, Region Ostdeutschland (vgl. dazu Tab. 2.A und Tab.3.A im Anhang).

Die Ergebnisse in den Mischberufen lassen zwei Schlussfolgerungen zu. Die Nivellierung des GPG mit zunehmendem NOG zugunsten der Frauen- und zuungunsten der Männerlöhne lässt sich einerseits vermutlich durch die allgemeine, die Lohnungleichheit nivellierende Wirkung von gewerkschaftlicher Organisation zurückführen. Die Inklusionsthese bestätigt sich damit. Auf der anderen Seite kann man davon ausgehen, dass Männer in den Mischberufen mit vergleichsweise niedrigem NOG, wie den Berufen in der Finanzdienstleistungsbranche, höhere Löhne erzielen als Frauen (vgl. Schröder \& Schäfer, 2013). 
Abb. 1: Zusammenhang von gewerkschaftlichem Nettoorganisationgrad und Stundenlohn für Männer und Frauen nach Berufstypen, vorhergesagte Werte
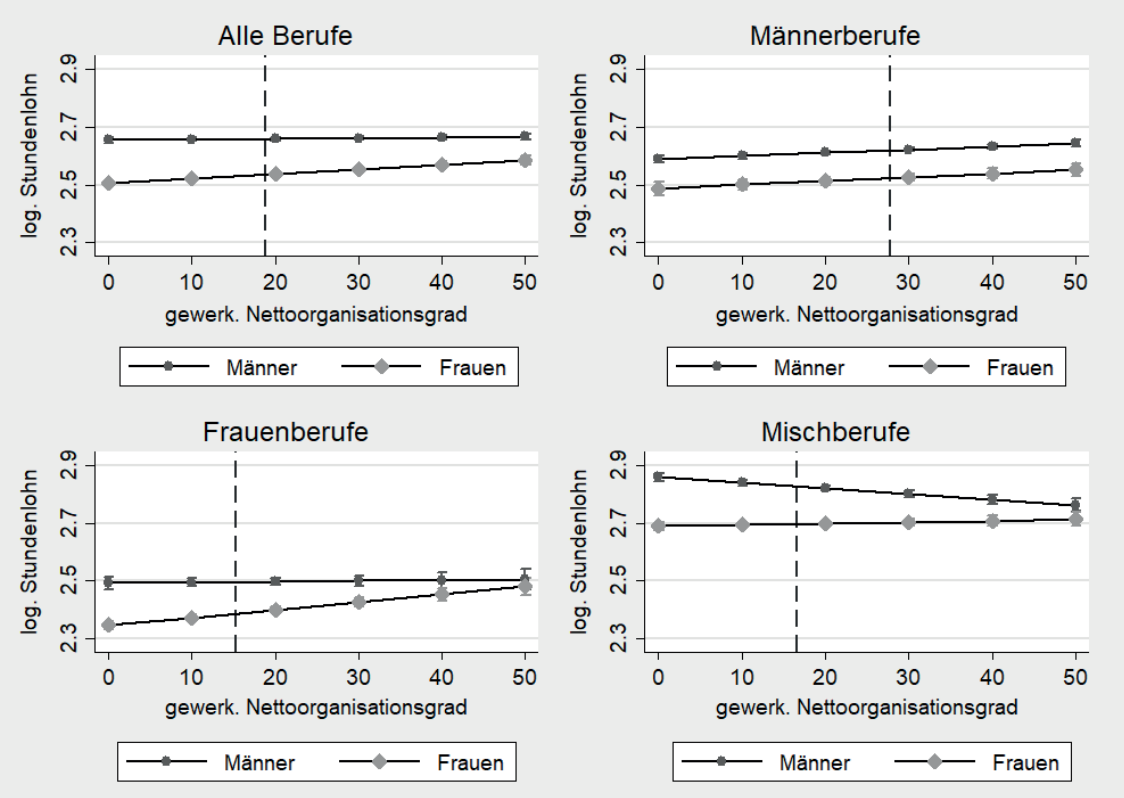

Anmerkung: Die vorhergesagten Werte des Stundenlohnes für verschiedene NOG ergeben sich aus der Schätzung der Modelle M3-M6, also nach Einbezug aller Kontrollvariablen, und wurden jeweils für Männer und Frauen berechnet. Die Steigungen der Geraden lassen sich an den jeweiligen Koeffizienten der Interaktionsterme von NOG und Geschlecht ablesen. Die gestrichelte vertikale Linie gibt den jeweiligen durchschnittlichen NOG an. Quelle: SOEPv30 (2001-2012), eigene Berechnungen

\section{Diskussion und Fazit}

Ziel des Beitrags war es, den Zusammenhang von gewerkschaftlichem Organisationsgrad und dem GPG in geschlechtersegregierten Berufen zu beleuchten. Konkret wurde der Frage nachgegangen, ob und in welchen Berufstypen - Männer-, Frauen- oder Mischberufen - Gewerkschaften zur Inklusion von Frauen und somit zur Reduzierung des GPG beitragen oder ob von Schließungsstrategien auszugehen ist, welche den GPG erhöhen.

Zunächst ist anzumerken, dass der GPG auch nach Kontrolle individueller Humankapitalausstattung sowie weiterer struktureller Beschäftigungsmerkmale weiterhin erhalten bleibt und in den Mischberufen am höchsten ausfällt. Dies könnte zwar auf nicht berücksichtigte, unbeobachtete Merkmale zurückzuführen sein, kann aber auch als eine allgemeine Benachteiligung von Frauen in den Lohnerträgen ihres Humankapitals interpretiert werden. Die Ergebnisse deuten ferner darauf hin, dass Gewerkschaften ihren Organisationsgrad als Machtressource nutzen können, um im Sinne einer Inklusionsstrategie diese Benachteiligung von Frauen zu einem Teil aufzuheben (vgl. Dingeldey, Schröder \& Kathmann, 2015). Dieser Befund widerspricht zunächst der Annahme, dass mit der traditionell männlich geprägten gewerkschaftlichen Organisation Prozesse sozialer Schließung männlicher Beschäftigter gegenüber Frauen zum Tragen kommen. 
Die differenzierte Betrachtung nach Männer-, Frauen- und Mischberufen ermöglicht einen tieferen Einblick in den Mechanismus der Lohnfindung in geschlechtersegregierten Berufen. Damit wurde die Frage beleuchtet, inwieweit Gewerkschaften ihre Machtressourcen geschlechtsspezifisch in unterschiedlichem Maße bündeln (können). Die hierzu auf Basis theoretischer Ansätze zur Geschlechtersegregation formulierten Thesen zur sozialen Schließung müssen auch hier verworfen werden. In den Frauen- und Mischberufen sinkt der GPG mit Erhöhung des NOG. In den Frauenberufen geschieht dies durch eine Anhebung des Lohnniveaus der Frauen. Die weiblich geprägten Gewerkschaften scheinen insofern ihre Machtressourcen zu bündeln und gegen die bestehende Ungleichheit zwischen den Geschlechtern einzusetzen. Die Männer hingegen können in den Frauenberufen ihre Löhne mit steigendem NOG nicht erhöhen. In den Mischberufen müssen sie sogar relative Lohnverluste in Kauf nehmen. Der hohe GPG kommt hier demnach vornehmlich dadurch zustande, dass Männer in Berufen und Branchen, in denen die Lohnungleichheit nicht durch gewerkschaftliche Organisation nivelliert wird, hohe Löhne erzielen können. Einzig in den Männerberufen steigen die Löhne mit dem NOG für beide Geschlechter gleichermaßen an, so dass sich der Niveau-Unterschied in den Löhnen nicht reduziert.

Ob Gewerkschaftsstrategien zum Tragen kommen, die - angesichts des Bedeutungsverlustes der Gewerkschaften in den 1990er und frühen 2000er Jahren - im Sinne des Organising (Briskin, 2011) explizit auf die historisch vernachlässigte Gruppe der Frauen gerichtet sind, kann aufgrund der gepoolten (zeitkonstanten) Daten zur Gewerkschaftsmitgliedschaft an dieser Stelle nicht beantwortet werden. Denkbar wäre auch, dass die Nivellierung des GPG in organisierten Berufsfeldern aus einer allgemeinen Reduktion der Lohnungleichheit resultiert, die Frauen in besonderem Maße strukturell begünstigt. Da der NOG der Frauen in allen Berufstypen (weit) unter dem der Männer liegt, liegt hier in jedem Fall noch erhebliches Mobilisierungspotential brach, worauf sich gewerkschaftliche Strategien richten könnten. Aufgabe zukünftiger Forschung sollte es daher sein, die hier festgestellte Inklusion von Frauen in der Lohndimension durch die Berücksichtigung spezifischer Merkmale der kollektiv organisierten und segregierten Berufe im Längsschnitt näher zu erklären.

\section{Literatur}

Achatz, D.-S. J., Gartner, H. \& Glück, T. (2005). Bonus oder Bias? Kölner Zeitschrift für Soziologie und Sozialpsychologie, 57(3), 466-493. https://doi.org/10.1007/s11577-005-0185-6

Acker, J. (1991). Thinking about wages: The gendered wage gap in Swedish banks. Gender and Society, 5(3), 390-407. https://doi.org/10.1177/089124391005003008

Acker, J. (1992). From sex roles to gendered institutions. Contemporary Sociology, 21(5), 565-569. https://doi.org/10.2307/2075528

Addison, J., Teixeira, P., Evers, K. \& Bellmann, L. (2014). Indicative and updated estimates of the collective bargaining premium in Germany. Industrial Relations: A Journal of Economy and Society, 53(1), 125-156. https://doi.org/10.1111/irel.12049

Aisenbrey, S. \& Brückner, H. (2008). Occupational aspirations and the gender gap in wages. European Sociological Review, 24(5), 633-649. https://doi.org/10.1093/esr/jen024

Amlinger, M. (2014). Lohnhöhe und Tarifbindung. Bestimmungsfaktoren der individuellen Verdiensthöhe (WSI Report No. 20). Düsseldorf.

Amlinger, M. \& Bispinck, R. (2013). Tarifbindung in Deutschland Ergebnisse der Verdienststrukturerhebung (VSE) (WSI Arbeitspapier No. 1). Düsseldorf. 
Antonczyk, D., Fitzenberger, B. \& Sommerfeld, K. (2010). Rising wage inequality, the decline of collective bargaining, and the gender wage gap. Labour Economics, 17(5), 835-847. https://doi.org/10.1016/j.labeco.2010.04.008

Arbeitskreis Strategic Unionism (2013). Jenaer Machtressourcenansatz 2.0. In S. Schmalz \& K. Dörre (Hg.), Comeback der Gewerkschaften? Machtressourcen, innovative Praktiken, internationale Perspektiven (pp. 345-375). Frankfurt/New York: Campus Verlag.

Beblo, M. \& Wolf, E. (2003). Sind es die Erwerbsunterbrechungen? Ein Erklärungsbeitrag zum Lohnunterschied zwischen Frauen und Männern in Deutschland. MittAB, 4, 560-572.

Biebeler, H. (2013). Mitgliederstruktur. Die Jüngeren holen auf. Gewerkschaftsspiegel, 2 , 1.

Bispinck, R. \& Schulten, Th. (2011): Das Tarifsystem stabilisieren - wie soll das gehen? Magazin Mitbestimmung, 7+8/2011, 27-30.

Blau, F.D. \& Kahn, L.M. (2003). Understanding International Differences in the Gender Pay Gap. Journal of Labor Economics, 21, 106-144. https://doi.org/10.1086/344125

Bol, T. \& Weeden, K. A. (2015). Occupational closure and wage inequality in Germany and the United Kingdom. European Sociological Review, 31(3), 354-369. https://doi.org/10.1093/esr/jcu095

Briskin, L. (2011). Union renewal, postheroic leadership, and women's organizing. Crossing discourses, reframing debates. Labor Studies Journal, 36(4), 508-537. https://doi.org/ $10.1177 / 0160449 \times 11422608$

Bryson, A. (2007). The effect of trade unions on wages. Reflets et perspectives de la vie économique, 46(2), 33-45. https://doi.org/10.3917/rpve.462.0033

Budig, M. J. (2002). Male advantage and the gender composition of jobs: Who rides the glass escalator? Social Problems, 49(2), 258-277. https://doi.org/10.1525/sp.2002.49.2.258

Busch, A. (2013). Der Einfluss der beruflichen Geschlechtersegregation auf den „Gender Pay Gap“. KZfSS Kölner Zeitschrift für Soziologie und Sozialpsychologie, 65(2), 301-338. https://doi. org/10.1007/s11577-013-0201-1

Carl, A.-H. \& Krehnke, A. (2004). Geschlechterdiskriminierung bei der betrieblichen Grundentgeltfindung. Wiesbaden: Deutscher Universitäts-Verlag. https://doi.org/10.1007/978-3-322-81737-2

Cho, D. \& Cho, J. (2011). How do labor unions influence the gender earnings gap? A comparative study of the US and Korea. Feminist economics, 17(3), 133-157. https://doi.org/10.1080/13545701.20 11.582472

Christofides, L.N., Polycarpou, A. \& Konstantinos,V.(2013). Gender wage gaps, 'sticky floors' and 'glass ceilings' in Europe. Labour Economics, 21, 86-102. https://doi.org/10.1016/j.labeco.2013.01.003

Dingeldey, I., Schröder, T. \& Kathmann, T. (2015). Zum Zusammenhang von prekären Beschäftigungsbedingungen und Interessenvertretung im Dienstleistungssektor. Industrielle Beziehungen, $22(3 / 4), 240-259$.

Ebbinghaus, B. \& Kittel, B. (2006). Europäische Sozialmodelle à la carte: Gibt es institutionelle Wahlverwandtschaften zwischen Wohlfahrtsstaat und Arbeitsbeziehungen? In Beckert, J. \& Ebbinghaus, B. \& Hassel, A. \& Manow, Ph. (Hg.), Transformationen des Kapitalismus. Festschrift für Wolfgang Streeck zum sechzigsten Geburtstag (pp. 223-246). Frankfurt/M.: Campus.

Elvira, M.M. \& Saporta, I. (2001). How does collective bargaining affect the gender pay gap? Work and Occupations, 28, 469-90. https://doi.org/10.1177/0730888401028004005

England, P. (2007). Devaluation and the pay of comparable male and female occupations. In D. B. Grusky \& S. Szelénye (Eds.), The inequality reader (pp. 352-356). Boulder, Colorado: Westview.

Even, W.E. \& Macphersen D.A. (1993). The decline of private-sector unionism and the gender wage gap. The Journal of Human Resources, 28(2), 279-296. https://doi.org/10.2307/146204

Fitzenberger, B. \& Kohn, K. (2005). Gleicher Lohn für gleiche Arbeit? Zum Zusammenhang zwischen Gewerkschaftsmitgliedschaft und Lohnstruktur in Westdeutschland 1985-1997. Zeitschrift für ArbeitsmarktForschung-Journal for Labour Market Research, 38(2/3), 125-146.

Fitzenberger, B., Kohn, K. \& Lembcke, A. C. (2013). Union density and varieties of coverage: The anatomy of union wage effects in Germany. Industrial \& Labor Relations Review, 66(1), 169-197. https://doi.org/10.1177/001979391306600107 
Friedmann. P. \& Pfau, B. (1985). Frauenarbeit in der Krise - Frauenarbeit trotz Krise? Korrekturversuch an einem arbeitsmarkttheoretischen Allgemeinplatz. Leviathan, 13(2), 155-186.

Gartner, H. (2016). Löhne von Frauen und Männern (IAB Aktuelle Berichte No. 7/2016).

Gärtner, D., Grimm, V., Lang, J., Stephan, G., Daumann, V., Dony, E., Knapp, B. \& Strien, K. (2014). Kollektive Lohnverhandlungen und der Gender Wage Gap: Befunde aus einer qualitativen Studie (IAB discussion paper, 14/2014).

Gerlach, K. \& Stephan, G. (2005). Tarifverträge und betriebliche Entlohnungsstrukturen (IAB discussion paper 20/2005)

Giesselmann, M. \& Windzio,M. (2012). Regressionsmodelle zur Analyse von Paneldaten. Wiesbaden: Springer VS.

Gottschall, K. \& Schröder, T. Familienlohn - Zur Entstehung einer wirkmächtigen Normierung geschlechtsspezifischer Arbeitsteilung. WSI-Mitteilungen, 03/2013, 161-170.

Hausmann, A.-C., Kleinert, C. \& Leuze, K. (2015). „Entwertung von Frauenberufen oder Entwertung von Frauen im Beruf?" Eine Längsschnittanalyse zum Zusammenhang von beruflicher Geschlechtersegregation und Lohnentwicklung in Westdeutschland. Kölner Zeitschrift für Soziologie, 67, 217-242. https://doi.org/10.1007/s11577-015-0304-y

Heinze, A. \& Wolf, E. (2010). The intra-firm gender wage gap: a new view on wage differentials based on linked employer-employee data. Journal of Population Economics, 23(3), 851-879. https://doi. org/10.1007/s00148-008-0229-0

Hirsch, B. \& Schnabel, C. (2014). What can we learn from bargaining models about union power? The decline of union power in Germany, 1992-2009. The Manchester School, 82(3), 347-362.

Hirsch, B., Oberfichtner, M. \& Schnabel,C. (2014). The levelling effect of product market competition on gender wage discrimination. IZA Journal of Labor Economics,19(3), 1-14. https://doi. org/10.1186/s40172-014-0013-1

Hox, J. (2002). Multilevel analysis: Techniques and applications. Mahwah: Lawrence Erlbaum.

Kanter, R. M. (1977). Some effects of proportions on group life: Skewed sex ratios and responses to token women. The American Journal of Sociology, 82(5), 965-990. https://doi.org/10.1086/226425

Kaufman, R. L. (2002). Assessing alternative perspectives on race and sex employment segregation. American Sociological Review, 67(4), 547-572. Stable URL: http://www.jstor.org/stable/3088945

Kleinert, C. \& Matthes, B. (2008). Educational expansion, segregation and occupational placement of women and men - Gender specific changes in entry jobs' prestige and wages. In A. Hadjar \& R. Becker (Eds.), Expected and unexpected consequences of the educational expansion in Europe and the US. Theoretical approaches and empirical findings in comparative perspective (pp. $327-$ 346). Bern: Haupt.

Kray, L. J. \& Thompson, L. (2004). Gender stereotypes and negotiation performance: An examination of theory and research. Research in organizational behavior, 26, 103-182. https://doi.org/10.1016/ S0191-3085(04)26004-X

Kunze, A. (2005). The evolution of the gender wage gap. Labour Economics, 12(1), 73-97. https://doi. org/10.1016/j.labeco.2004.02.012

Liebeskind, U. (2004). Arbeitsmarktsegregation und Einkommen. Vom Wert „weiblicher“ Arbeit. Kölner Zeitschrift für Soziologie und Sozialpsychologie, 56, 630-652. doi:10.1007/s11577-004$0107-\mathrm{z}$

Luhmann, N. (1997). Die Gesellschaft der Gesellschaft. Frankfurt am Main: Suhrkamp.

Mazei, J., Hüffmeier, J., Freund, P. A., Stuhlmacher, A. F., Bilke, L. \& Hertel, G. (2015). A meta-analysis on gender differences in negotiation outcomes and their moderators. Psychological bulletin, 141(1), 85. https://doi.org/10.1111/j.1744-6570.1999.tb00175.x

McGuinness, S., Kelly, E., O'Connell, P.J. \& Callan, T. (2011). The impact of wage bargaining and worker preferences on the gender pay gap. European Journal of Industrial Relations, 17(3), 277 293. https://doi.org/10.1177/0959680111410962

Mincer, J. (1974). Schooling, experience, and earnings. New York: NBER.

Offe, C. \& Hinrichs, K. (1977). Sozialökonomie des Arbeitsmarktes und die Lage benachteiligter Gruppen von Arbeitnehmern. In C. Offe (Ed.), Opfer des Arbeitsmarktes: Zur Theorie der strukturierten 
Arbeitslosigkeit - Kritische Texte: Sozialarbeit, Sozialpädagogik, soziale Probleme. (pp. 3-61). Neuwied/Darmstadt: Luchterhand Verlag.

Parkin, F. (1979). Marxism and Class Theory: A Bourgeois Critique. New York: Tavistock Publications.

Pries, L. (2010). Erwerbsregulierung in einer globalisierten Welt. Wiesbaden: VS Verlag für Sozialwissenschaften. https://doi.org/10.1007/978-3-531-91956-0

Rabe-Hesketh, S. \& Skrondal, A. (2012). Multilevel and longitudinal modeling using stata. Volume I: Continuous Responses. 3rd edition. College Station, Texas: Stata Press.

Schäfer, A. \& Gottschall, K. (2015). From wage regulation to wage gap: how wage-setting institutions and structures shape the gender wage gap across three industries in 24 European countries and Germany. Cambridge Journal of Economics, 39, 467-496. https://doi.org/10.1093/cje/bev005

Schmalz, S. \& Dörre, K. (2014). Der Machtressourcenansatz: Ein Instrument zur Analyse gewerkschaftlichen Handlungsvermögens. Industrielle Beziehungen, 21(3), 217-237. https://doi.org/10.1688/ IndB-2014-03-Schmalz

Schmierl, K. (2003). Wird das deutsche Modell der Arbeitsregulierung die Umschichtungen in der Arbeitsgesellschaft überleben? WSI Mitteilungen, 11/2003, 651-658.

Schnabel, C. (2005). Gewerkschaften und Arbeitgeberverbände: Organisationsgrade, Tarifbindung und Einflüsse auf Löhne und Beschäftigung. Zeitschrift für Arbeitsmarktforschung, 38(2/3), 181-196.

Schröder, T. \& Schäfer, A. (2013). Wer erhält einen Ernährerlohn? Befunde nach Region und Geschlecht. WSI-Mitteilungen Schwerpunktheft, 3, 171-181.

Schröder, T., Struck,O. \& Wlodarski,C. (2008). Vordringlichkeit des Befristeten? Zur Theorie und Empirie offener Beschäftigungssysteme. In Köhler,C., Struck,O., Grotheer,M., Krause, A., Krause, I. \& Schröder,T. (Eds.): Offene und geschlossene Beschäftigungssysteme (pp. 143-200). Wiesbaden. VS Verlag für Sozialwissenschaften. https://doi.org/10.1007/978-3-531-91117-5_5

Stuhlmacher, A. F. \& Walters, A. E. (1999). Gender differences in negotiation outcome: A meta-analysis. Personnel Psychology, 52(3), 653-677. https://doi.org/10.1111/j.1744-6570.1999.tb00175.x

Stuth, S. \& Henning, M. (2014). Ist der Beruf entscheidend? Zum Einfluss beruflicher Eigenschaften auf die Dauer familienbedingter Nichterwerbsphasen von Frauen. (WZB discussion paper No. P2014-006). Berlin.

Treiman, D. J. \& Hartmann, H. I. (1981). Women, work, and wages: Equal pay for jobs of equal value. Washington, D.C.: National Academy Press. https://doi.org/10.17226/91

Weber, M. (1921[1976]). Wirtschaft und Gesellschaft. Grundriss der verstehenden Soziologie. Tübingen: Mohr.

Weeden, K. A. \& Sørensen, J. B. (2004). A framework for analyzing industrial and occupational sex segregation. In M. Charles \& D. B. Grusky (Eds.), Occupational ghettos. The worldwide segregation of women and men (pp. 245-294). Stanford: Stanford University Press.

Wieber, A. \& Holst, E. (2015). Gender identity and women's supply of labor and non-market eork: Panel data evidence for Germany. DIW Berlin Discussion Paper No. 1517. https://doi.org/10.2139/ ssrn. 2688965

Williams, C. (1992). The glass escalator: Hidden advantages for men in the "female" professions. Social Problems, 39(3), 253-267. https://doi.org/10.2307/3096961

Witz, A. (1990). Patriarchy and the Professions: The Gendered Politics of Occupational Closure. Sociology, 24(4), 675-690. https://doi.org/10.1177/0038038590024004007

Wunnava, P.V.\& Ewing, B.T. (2000). Union-Nonunion gender wage and benefit differentials across establishment sizes. Small Business Economics, 15, 47-57. https://doi.org/10.1023/A:1026593429470

Yoder, J. D. (1991). Rethinking tokenism: Looking beyond numbers. Gender and Society, 5(2), 178192. https://doi.org/10.1177/089124391005002003 


\section{Anhang}

Tab. 1.A: Deskriptive Statistiken (Prozent / aMedian)

\begin{tabular}{|c|c|c|c|c|}
\hline Variable & Alle Berufe & $\begin{array}{r}\text { Männer- } \\
\text { berufe }\end{array}$ & $\begin{array}{l}\begin{array}{l}\text { Frauen- } \\
\text { berufe }\end{array} \\
\end{array}$ & $\begin{array}{l}\text { Misch- } \\
\text { berufe }\end{array}$ \\
\hline GPG & 22,9 & 15,5 & 24,7 & 19,8 \\
\hline Bruttostundenlohn in Euro & 14,0 & 13,9 & 16,8 & 11,6 \\
\hline Alter in Jahrena & 43,0 & 43,0 & 42,0 & 44,0 \\
\hline \multicolumn{5}{|l|}{ Familienstand } \\
\hline Verheiratet & 57,5 & 62,8 & 54,2 & 57,0 \\
\hline Nicht-eheliche Lebensgemeinschaft & 14,1 & 12,4 & 16,0 & 13,5 \\
\hline Single & 28,4 & 24,8 & 29,8 & 29,5 \\
\hline \multicolumn{5}{|l|}{ Kinder } \\
\hline keine Kinder & 66,9 & 61,7 & 69,7 & 68,1 \\
\hline ein Kind & 17,4 & 17,7 & 16,7 & 17,7 \\
\hline zwei Kinder & 12,9 & 15,3 & 11,4 & 12,7 \\
\hline drei und mehr Kinder & 2,8 & 5,2 & 2,3 & 1,5 \\
\hline \multicolumn{5}{|l|}{ Bildung } \\
\hline niedrige & 9,2 & 14,2 & 6,8 & 8,0 \\
\hline mittlere & 56,0 & 58,6 & 40,9 & 70,4 \\
\hline Hohe & 34,8 & 27,2 & 52,3 & 21,7 \\
\hline Berufsprestige nach Treiman (SIOPS)a & 44,3 & 40,1 & 50,1 & 40,8 \\
\hline Dauer der Vollzeitberufserfahrung in Monatena & 138,0 & 174,0 & 141,3 & 98,7 \\
\hline Dauer der Betriebszugehörigkeit in Jahrena & 9,1 & 12,8 & 11,9 & 10,6 \\
\hline \multicolumn{5}{|l|}{ Arbeitsumfang } \\
\hline Vollzeit & 73,2 & 91,8 & 78,6 & 53,2 \\
\hline Teilzeit & 20,7 & 4,3 & 18,1 & 36,0 \\
\hline geringfügig /unregelmäßig & 6,1 & 3,9 & 3,3 & 10,8 \\
\hline Überstunden pro Woche a & 2,3 & 2,4 & 2,8 & 1,8 \\
\hline \multicolumn{5}{|l|}{ Unternehmensgröße } \\
\hline bis unter 20 & 22,6 & 19,4 & 17,5 & 30,6 \\
\hline 20 bis unter 200 & 29,9 & 30,4 & 28,5 & 31,0 \\
\hline 200 bis unter 2000 & 24,5 & 22,2 & 27,7 & 22,8 \\
\hline \multirow{2}{*}{\multicolumn{5}{|c|}{ Region }} \\
\hline & & & & \\
\hline West & 83,8 & 83,1 & 83,8 & 84,5 \\
\hline Ost & 16,2 & 17,0 & 16,2 & 15,5 \\
\hline \multicolumn{5}{|l|}{ Geschlecht } \\
\hline Mann & 50,5 & 88,0 & 49,6 & 19,6 \\
\hline Frau & 49,5 & 12,0 & 50,4 & 80,4 \\
\hline \multicolumn{5}{|l|}{ Berufstyp } \\
\hline Männerberuf & 27,1 & & & \\
\hline Mischberuf & 37,9 & & & \\
\hline Frauenberuf & 35,1 & & & \\
\hline $\begin{array}{l}\text { NOG nach Branchen-Berufs-Zellen } \\
\text { (davon Frauen) }\end{array}$ & $18,9(34,9)$ & $\begin{array}{l}27,8 \\
(7,8) \\
\end{array}$ & $\begin{array}{r}16,6 \\
(40,6) \\
\end{array}$ & $\begin{array}{r}15,5 \\
(68,8) \\
\end{array}$ \\
\hline $\mathrm{N}$ & 45.698 & 12.481 & 17.762 & 15.455 \\
\hline
\end{tabular}

Anmerkung: a Median

Quelle: SOEPv30 (2001-2012), eigene, gewichtete Berechnungen 
Tab. 2.A: Drei-Ebenenmodelle logarithmierter Bruttostundenlohn (alle Berufe)

\begin{tabular}{|c|c|c|c|}
\hline & Modell 1 & Modell 2 & Modell 3 \\
\hline \multicolumn{4}{|l|}{ Familienstand (Ref.: verheiratet) } \\
\hline Nichteheliche Lebensgemeinschaft & $\begin{array}{l}-0.009^{*} \\
(0.004)\end{array}$ & $\begin{array}{l}-0.009^{*} \\
(0.004)\end{array}$ & $\begin{array}{l}-0.009^{*} \\
(0.004)\end{array}$ \\
\hline Single & $\begin{array}{l}-0.044^{+* *+} \\
(0.004)\end{array}$ & $\begin{array}{l}-0.044^{\prime * *+} \\
(0.004)\end{array}$ & $\begin{array}{l}-0.044^{+\ldots+4} \\
(0.004)\end{array}$ \\
\hline Alter & $\begin{array}{c}0.004^{* * *} \\
(0.000)\end{array}$ & $\begin{array}{l}0.004^{* * *} \\
(0.000)\end{array}$ & $\begin{array}{l}0.004^{* * *} \\
(0.000)\end{array}$ \\
\hline \multicolumn{4}{|l|}{ Anzahl der Kinder (Ref.: keine) } \\
\hline Ein Kind & $\begin{array}{c}0.054^{* * *} \\
(0.004)\end{array}$ & $\begin{array}{l}0.054^{\text {tot }} \\
(0.004)\end{array}$ & $\begin{array}{l}0.054^{\text {t*t* }} \\
(0.004)\end{array}$ \\
\hline Zwei Kinder & $\begin{array}{c}0.088^{* * *} \\
(0.004)\end{array}$ & $\begin{array}{l}0.088^{+1+4} \\
(0.004)\end{array}$ & $\begin{array}{l}0.088^{+1 *} \\
(0.004)\end{array}$ \\
\hline Drei und mehr Kinder & $\begin{array}{c}0.107^{* * *} \\
(0.007)\end{array}$ & $\begin{array}{l}0.107^{+\ldots+1} \\
(0.007)\end{array}$ & $\begin{array}{l}0.107^{+\ldots+*} \\
(0.007)\end{array}$ \\
\hline \multicolumn{4}{|l|}{ Bildung (Ref.: niedrige) } \\
\hline Mittlere & $\begin{array}{c}0.061^{* * *} \\
(0.005)\end{array}$ & $\begin{array}{l}0.061^{\text {th+ }} \\
(0.005)\end{array}$ & $\begin{array}{l}0.061^{* * *} \\
(0.005)\end{array}$ \\
\hline Hohe & $\begin{array}{l}0.185^{* * *} \\
(0.005)\end{array}$ & $\begin{array}{l}0.186^{\ldots+1} \\
(0.005)\end{array}$ & $\begin{array}{l}0.186^{* \ldots} \\
(0.005)\end{array}$ \\
\hline Berufsprestige nach Treiman & $\begin{array}{l}0.010^{* * *} \\
(0.000)\end{array}$ & $\begin{array}{l}0.010^{* * *} \\
(0.000)\end{array}$ & $\begin{array}{l}0.010^{* \star *} \\
(0.000)\end{array}$ \\
\hline $\begin{array}{l}\text { Dauer der Vollzeitberufserfahrung in Monaten } \\
\text { Umfang der Erwerbstätigkeit (Ref.: Vollzeit) }\end{array}$ & $\begin{array}{l}0.000^{* * *} \\
(0.000)\end{array}$ & $\begin{array}{c}0.000^{* * *} \\
(0.000)\end{array}$ & $\begin{array}{l}0.000^{* * *} \\
(0.000)\end{array}$ \\
\hline Teilzeit & $\begin{array}{c}-0.059^{* * *} \\
(0.004)\end{array}$ & $\begin{array}{l}-0.059^{* * *+} \\
(0.004)\end{array}$ & $\begin{array}{l}-0.059^{+* *+} \\
(0.004)\end{array}$ \\
\hline Geringfügig/unregelmäßig & $\begin{array}{c}-0.305^{* * *} \\
(0.007)\end{array}$ & $\begin{array}{c}-0.304^{* *+*} \\
(0.007)\end{array}$ & $\begin{array}{l}-0.303^{+* *} \\
(0.007)\end{array}$ \\
\hline Öffentlicher Dienst & $\begin{array}{c}0.014^{* * *} \\
(0.004)\end{array}$ & $\begin{array}{l}0.013^{*} \\
(0.004)\end{array}$ & $\begin{array}{l}0.013^{*} \\
(0.004)\end{array}$ \\
\hline Dauer der Berufszugehörigkeit in Jahren & $\begin{array}{c}0.007^{* \star *} \\
(0.000)\end{array}$ & $\begin{array}{c}0.007^{* * *} \\
(0.000)\end{array}$ & $\begin{array}{c}0.007^{\star * *} \\
(0.000)\end{array}$ \\
\hline Überstunden & $\begin{array}{c}-0.003^{* * *} \\
(0.000)\end{array}$ & $\begin{array}{c}-0.003^{* * *} \\
(0.000)\end{array}$ & $\begin{array}{c}-0.003^{* * *} \\
(0.000)\end{array}$ \\
\hline \multicolumn{4}{|l|}{ Region (Ref.: Westdeutschland) } \\
\hline Ostdeutschland & $\begin{array}{c}-0.268^{* * *} \\
(0.003)\end{array}$ & $\begin{array}{l}-0.268^{+*+1} \\
(0.003)\end{array}$ & $\begin{array}{l}-0.268^{+1+2} \\
(0.003)\end{array}$ \\
\hline Frau & $\begin{array}{c}-0.124^{\star * *} \\
(0.004)\end{array}$ & $\begin{array}{l}-0.124^{+3+x} \\
(0.004)\end{array}$ & $\begin{array}{c}-0.149^{+1+2} \\
(0.006)\end{array}$ \\
\hline NOG nach Branchen-Berufs-Zellen & & $\begin{array}{l}0.007^{* \star *} \\
(0.002)\end{array}$ & $\begin{array}{c}0.002 \\
(0.002)\end{array}$ \\
\hline Frau*NOG nach Branchen-Berufs-Zellen & & & $\begin{array}{l}0.014^{+*+*} \\
(0.002)\end{array}$ \\
\hline \multicolumn{4}{|l|}{ Unternehmensgröße (Ref. unter 20) } \\
\hline $20-200$ & $\begin{array}{c}0.104^{* * *} \\
(0.004)\end{array}$ & $\begin{array}{l}0.103^{* * *} \\
(0.004)\end{array}$ & $\begin{array}{l}0.103^{* * *} \\
(0.004)\end{array}$ \\
\hline $200-2000$ & $\begin{array}{l}0.178^{* * *} \\
(0.004)\end{array}$ & $\begin{array}{l}0.176^{* * *} \\
(0.004)\end{array}$ & $\begin{array}{l}0.176^{* * *} \\
(0.004)\end{array}$ \\
\hline Mehr als 2000 & $\begin{array}{l}0.231^{* * *} \\
(0.004)\end{array}$ & $\begin{array}{l}0.229^{\star \star \star} \\
(0.004)\end{array}$ & $\begin{array}{l}0.229^{* * *} \\
(0.004)\end{array}$ \\
\hline \multicolumn{4}{|l|}{ Berufstyp (Ref.: Männerberuf) } \\
\hline Mischberuf & $\begin{array}{c}0.071^{* * *} \\
(0.006)\end{array}$ & $\begin{array}{l}0.077^{+*+1} \\
(0.007)\end{array}$ & $\begin{array}{l}0.075^{\text {t***}} \\
(0.007)\end{array}$ \\
\hline Frauenberuf & $\begin{array}{l}-0.016^{*} \\
(0.007)\end{array}$ & $\begin{array}{l}-0.009 \\
(0.008)\end{array}$ & $\begin{array}{l}-0.007 \\
(0.008)\end{array}$ \\
\hline Konstante & $\begin{array}{l}1.675^{* * *} \\
(0.015)\end{array}$ & $\begin{array}{l}1.657^{+*+1} \\
(0.016)\end{array}$ & $\begin{array}{l}1.668^{+1+*} \\
(0.016)\end{array}$ \\
\hline Var (Jahre) & 0,000 & 0,000 & 0,0000 \\
\hline Var (Berufe) & 0,0115 & 0,0115 & 0,0115 \\
\hline Residualvarianz & 0,1297 & 0,1300 & 0,1355 \\
\hline $\mathrm{AIC} / \mathrm{BIC}$ & $67040 / 67384$ & $67030 / 67383$ & $70560 / 70922$ \\
\hline $\mathrm{N}$ & 80.718 & 80.718 & 80.718 \\
\hline
\end{tabular}

Anmerkungen: * $p<0.05,{ }^{* *} p<0.01,{ }^{* * *} p<0.001$, Standardfehler in Klammern Quelle: SOEPv30 (2001-2012), eigene Berechnungen 
Tab. 3.A: Drei-Ebenenmodelle logarithmierter Bruttostundenlohn (Berufstypen)

\begin{tabular}{|c|c|c|c|}
\hline & Männerberufe (M4) & Frauenberufe (M5) & Mischberufe (M6) \\
\hline Alter & $\begin{array}{l}0.002^{* \star *} \\
(0.000)\end{array}$ & $\begin{array}{l}0.002^{* * *} \\
(0.000)\end{array}$ & $\begin{array}{c}0.006^{* * *} \\
(0.000)\end{array}$ \\
\hline \multicolumn{4}{|l|}{ Familienstand (Ref.: Verheiratet) } \\
\hline Nichteheliche Lebensgemeinschaft & $\begin{array}{l}-0.031^{*+*+} \\
(0.007)\end{array}$ & $\begin{array}{l}0.027^{7+*} \\
(0.008)\end{array}$ & $\begin{array}{l}-0.015^{*} \\
(0.007)\end{array}$ \\
\hline Single & $\begin{array}{l}-0.060^{*+*} \\
(0.006)\end{array}$ & $\begin{array}{l}-0.025^{* * *} \\
(0.006)\end{array}$ & $\begin{array}{l}-0.046^{+* *+} \\
(0.006)\end{array}$ \\
\hline \multicolumn{4}{|l|}{ Anzahl der Kinder (Ref.: keine) } \\
\hline Ein Kind & $\begin{array}{l}0.027^{+1+*} \\
(0.006)\end{array}$ & $\begin{array}{l}0.038^{*+*} \\
(0.006)\end{array}$ & $\begin{array}{l}0.077^{+* *} \\
(0.006)\end{array}$ \\
\hline Zwei Kinder & $\begin{array}{l}0.055^{+1+} \\
(0.006)\end{array}$ & $\begin{array}{l}0.074^{*+*} \\
(0.008)\end{array}$ & $\begin{array}{l}0.115^{+* *+} \\
(0.007)\end{array}$ \\
\hline Drei und mehr Kinder & $\begin{array}{l}0.044^{+1+4} \\
(0.010)\end{array}$ & $\begin{array}{l}0.119^{*+1} \\
(0.015)\end{array}$ & $\begin{array}{l}0.138^{+1} \\
(0.012)\end{array}$ \\
\hline \multicolumn{4}{|l|}{ Bildung (Ref.: niedrige) } \\
\hline Mittlere & $\begin{array}{l}0.045^{\text {t+* }} \\
(0.007)\end{array}$ & $\begin{array}{l}0.061^{+* *+} \\
(0.008)\end{array}$ & $\begin{array}{l}0.089^{*+*} \\
(0.009)\end{array}$ \\
\hline Hohe & $\begin{array}{l}0.128^{+1 *} \\
(0.008)\end{array}$ & $\begin{array}{l}0.144^{*+*} \\
(0.009)\end{array}$ & $\begin{array}{l}0.270^{\prime * *} \\
(0.010)\end{array}$ \\
\hline Berufsprestige nach Treiman & $\begin{array}{l}0.009^{* * *} \\
(0.000)\end{array}$ & $\begin{array}{l}0.010^{* * *} \\
(0.000)\end{array}$ & $\begin{array}{l}0.009^{* * *} \\
(0.000)\end{array}$ \\
\hline $\begin{array}{l}\text { Dauer der Vollzeitberufserfahrung } \\
\text { in Monaten }\end{array}$ & $\begin{array}{l}0.000^{* * *} \\
(0.000)\end{array}$ & $\begin{array}{l}0.000^{* * *} \\
(0.000)\end{array}$ & $\begin{array}{l}0.000^{* * *} \\
(0.000)\end{array}$ \\
\hline \multicolumn{4}{|l|}{ Umfang der Erwerbstätigkeit (Ref.: Vollzeit) } \\
\hline Teilzeit & $\begin{array}{l}-0.168^{*+*+} \\
(0.011)\end{array}$ & $\begin{array}{l}-0.0266^{* * *} \\
(0.007)\end{array}$ & $\begin{array}{l}-0.058^{+*+*} \\
(0.007)\end{array}$ \\
\hline Geringfügig/unregelmäßig & $\begin{array}{l}-0.392^{+*+} \\
(0.015)\end{array}$ & $\begin{array}{l}-0.220^{\prime *} \\
(0.010)\end{array}$ & $\begin{array}{l}-0.415^{\prime+} \\
(0.014)\end{array}$ \\
\hline $\begin{array}{l}\text { Dauer der Betriebszugehörigkeit in } \\
\text { Jahren }\end{array}$ & $0.007^{* * *}$ & $0.007^{* * *}$ & $0.007^{* * *}$ \\
\hline & $(0.000)$ & $(0.000)$ & $(0.000)$ \\
\hline Überstunden & $\begin{array}{c}-0.004^{* * *} \\
(0.001)\end{array}$ & $\begin{array}{c}-0.007^{* * *} \\
(0.001)\end{array}$ & $\begin{array}{c}-0.002^{* * *} \\
(0.001)\end{array}$ \\
\hline Öffentlicher Dienst & $\begin{array}{l}0.026^{*} \\
(0.008)\end{array}$ & $\begin{array}{l}0.053^{\prime+1+} \\
(0.007)\end{array}$ & $\begin{array}{l}-0.033^{\prime+1} \\
(0.007)\end{array}$ \\
\hline Region (Ref.: Westdeutschland) & & & \\
\hline Ostdeutschland & $\begin{array}{l}-0.301^{2+*+} \\
(0.005)\end{array}$ & $\begin{array}{l}-0.251^{m+*} \\
(0.006)\end{array}$ & $\begin{array}{c}-0.250^{\text {tht }} \\
(0.006)\end{array}$ \\
\hline Frau & $\begin{array}{l}-0.102^{+*+*} \\
(0.014)\end{array}$ & $\begin{array}{l}-0.147^{\prime+*} \\
(0.013)\end{array}$ & $\begin{array}{l}-0.170^{\prime+1+} \\
(0.009)\end{array}$ \\
\hline NOG nach Branchen-Berufs-Zellen & $\begin{array}{c}0.011^{* * *} \\
(0.002)\end{array}$ & $\begin{array}{c}0.002 \\
(0.006)\end{array}$ & $\begin{array}{c}-0.019^{* \star *} \\
(0.004)\end{array}$ \\
\hline Frau*NOG nach Branchen-Berufs-Zellen & $\begin{array}{c}0.002 \\
(0.005)\end{array}$ & $\begin{array}{l}0.024^{*+*} \\
(0.007)\end{array}$ & $\begin{array}{l}0.024^{*+1} \\
(0.004)\end{array}$ \\
\hline Unternehmensgröße (Ref. unter 20) & & & \\
\hline $20-200$ & $\begin{array}{c}0.080^{* * *} \\
(0.006)\end{array}$ & $\begin{array}{l}0.109^{* * *} \\
(0.006)\end{array}$ & $\begin{array}{l}0.111^{* * *} \\
(0.007)\end{array}$ \\
\hline $200-2000$ & $\begin{array}{l}0.181^{* * *} \\
(0.007)\end{array}$ & $\begin{array}{l}0.178^{* * *} \\
(0.007)\end{array}$ & $\begin{array}{l}0.167^{* * *} \\
(0.008)\end{array}$ \\
\hline Mehr als 2000 & $\begin{array}{c}0.258^{* * *} \\
(0.007)\end{array}$ & $\begin{array}{l}0.201^{* * *} \\
(0.008)\end{array}$ & $\begin{array}{c}0.220^{* \star *} \\
(0.007)\end{array}$ \\
\hline Konstante & $\begin{array}{l}1.813^{\text {th }} \\
(0.024)\end{array}$ & $\begin{array}{l}1.685^{5+1} \\
(0.029)\end{array}$ & $\begin{array}{l}1.640^{+1} \\
(0.028)\end{array}$ \\
\hline Var (Jahre) & 0,000 & 0,000 & 0,000 \\
\hline Var (Berufe) & 0,0103 & 0,0060 & 0,0140 \\
\hline Residualvarianz & 0,0969 & 0,1449 & 0,1345 \\
\hline $\mathrm{AIC} / \mathrm{BIC}$ & $13103 / 13401$ & $24743 / 25046$ & $26946 / 27254$ \\
\hline $\mathrm{N}$ & 23.646 & 26.702 & 30.370 \\
\hline
\end{tabular}

Anmerkungen: * $p<0.05,{ }^{* *} p<0.01,{ }^{* * *} p<0.001$, Standardfehler in Klammern

Quelle: SOEPv30 (2001-2012), eigene Berechnungen 


\section{Duisburg-Essen Publications online}

Dieser Text wird über DuEPublico, dem Dokumenten- und Publikationsserver der Universität Duisburg-Essen, zur Verfügung gestellt. Die hier veröffentlichte Version der EPublikation kann von einer eventuell ebenfalls veröffentlichten Verlagsversion abweichen.

DOI: $\quad$ 10.3224/indbez.v24i2.04

URN: urn:nbn:de:hbz:464-20200716-154658-1 